\title{
Soft Part 3D visualization by serial sectioning and computer reconstruction
}

\author{
BERNHARD RUTHENSTEINER \\ Bavarian State Collection of Zoology, Münchhausenstraße 21, 81247 Munich, Germany, \\ E-mail: BRuthensteiner@zsm.mwn.de
}

\begin{abstract}
Recent increases in performance of personal computer hard- and software enabled a variety of 3D graphical applications, such as surface visualizations of biological specimens. This indirectly recalled an old morphological method back to life, the investigation of soft part anatomy by light microscopical serial section analysis. A practical guide covering all procession steps beginning with anesthetization leading to the final goal, 3D visualization of specimens, is provided. Most useful for 3D procession —of particularly small specimens—are ribbon-forming serial resin (= "semithin") sections. A reliable method for achievement of ribbon formation is described in detail for the first time. Contact cement is applied only to the cutting surface of the block, which represents a modification of an old protocol. Details on the materials and tools, such as embedding media (epoxy resins) and knives (Ralph glass or special diamond knife) used and general handling for the entire procedure are given and critically evaluated. 3D procession is explained for the software $A M I R A^{\circledR}$. The major processing steps, from section image capturing until refining of surfaces, are explained. Based on the experience of the author, practical aids that cannot be found in the user's guide of the software or elsewhere for facilitating the process are given. These include preliminary calculation of resolution, calibration and strategies for facilitating the process and improvement of results. The interpolate function is emphasized as most useful for completion of segmentation and also correction works. Visualization examples are followed by an estimate of work expenditure for graphical processes involved.
\end{abstract}

Key words: Micromolluscs, methods, histology, serial sectioning, ribbon formation, 3D, reconstruction, visualization

\section{Introduction}

Examination of morphological structures by means of serial section analysis reaches back to the $19^{\text {th }}$ century. Originally this was performed by using paraffin for embedding, a method that basically remained unchanged until today. A major improvement to light optic serial section analysis resulted from the invention of transmission electron microscopy (=TEM), which was established as routine method in the 1950s to 1960s. The electron beam used thereby required thinner sections than for light microscopy, which had to be based on a more stable embedding medium. Synthetic resin proved to be the suitable embedding medium for that purpose and soon a variety of different resin kinds together with a new sectioning array (glass knives with floating sections on water) were successfully applied. As a side effect, it turned out that resin sections, if sectioned thicker ("semithin" (!) in contrast to "(ultra-)thin" sections) than for TEM, can be valuable for light microscopical purposes too. In the beginning analyses of resin sections by light microscopy were typically only applied in addition to a main method like TEM investigation. Later on, the merits for light microscopy, compared to conventional methods like paraffin sectioning, were recognized. They particularly regard two fields of application: (1) Because of similarities in texture between the chitin skeleton and the resin, sections 
show much fewer artifacts in arthropods (e.g., Pass 1985). (2) In small specimens the amount of information gained is substantially higher because of lesser section thickness and higher section quality (e.g., Haszprunar 1988).

The main problem of resin sectioning for a long time was that sections, unlike as for other sectioning techniques (e.g., paraffin sectioning, TEM sectioning), would not form ribbons during the cutting process. Sections, therefore, had to be manipulated separately. This led to a high expenditure of work in production of series, where sections mostly were transferred one by one to the slide. Furthermore, such series caused more effort and difficulties in analysis, as they were placed distantly to each other and unaligned regarding "rotation" on the slide (Fig. 1A). Consequently, there were repeated attempts - by coating the block or applying different substances - to achieve ribbon forming of such sections (see e.g., Reid \& Beesley 1991 and Blumer et al. 2002 for references). None of those, however, were really reliable. In the late 1980s I could overcome this problem and successfully establish a reliable way for obtaining ribbons by modifying a method suggested by Henry (1977) (Fig. 1B). This method, however, was not initially published as such, but only briefly and imprecisely mentioned in Material and Method parts of result-based papers (e.g., Ruthensteiner \& Schaefer 1991; Ruthensteiner 1997, 1999; Ruthensteiner et al. 2001). Later I was invited to share a co-authorship in a methodfocused paper by Blumer et al. (2002), which chiefly served to introduce the very useful commercially available Histo Jumbo diamond knife (see below for specifications), because it included "my" ribbon formation technique. However, I did not have the opportunity to revise all methodological details. This is why some information on handling given in that paper in my opinion is insufficient or misleading (e.g., block trimming, manipulation of ribbons, pre-treatment of slides).

Despite of the availability of ribbon formation, the "semithin" sectioning technique, just like other light-microscopical sectioning techniques, was increasingly relegated to the fringe in morphology. This primarily was due to the increasing dominance of other methods, such as immunocytochemical and histochemical staining of whole mounts followed by analysis with laser scanning confocal mi-

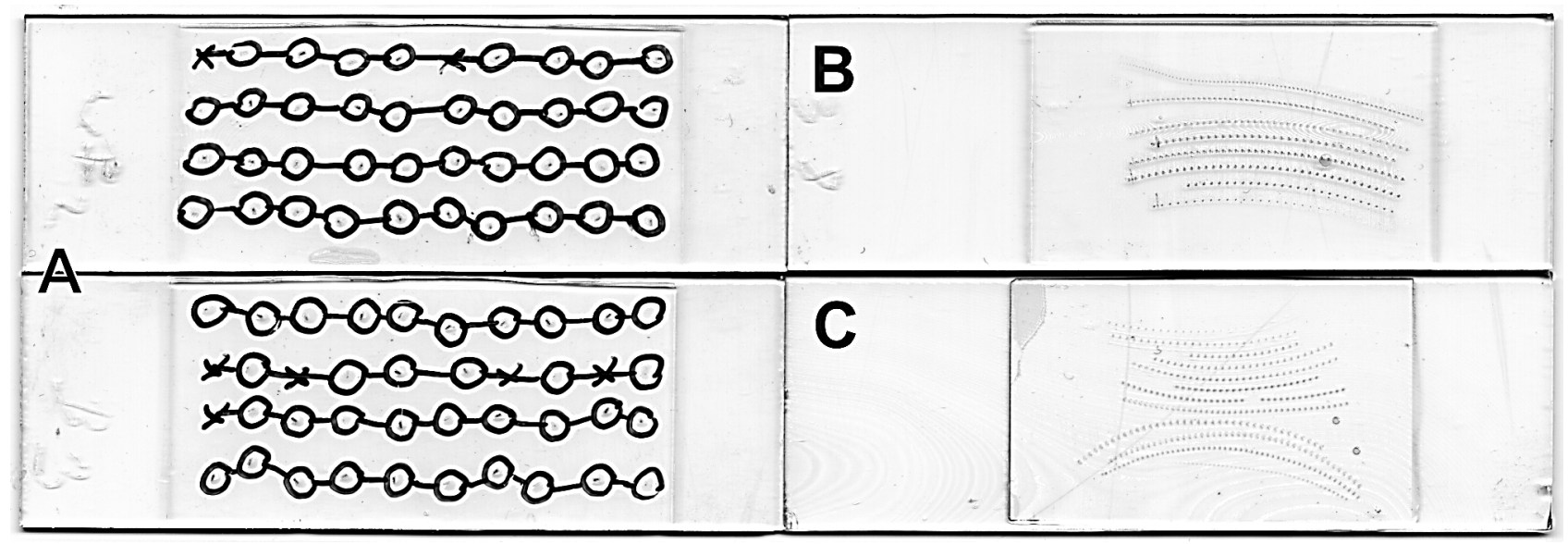

Figure 1. Comparison of the separate section method with ribbon forming sections. Single image taken with flatbed scanner. A. Example with two slides of a series (second and third of a total of eight) of separately placed sections cut at a thickness of $1 \mu \mathrm{m}$. Sections encircled and connected with line with permanent marker (underside of the slide to avoid dissolution with immersion oil) to facilitate tracing them at the microscope. B-C. Examples of complete series of specimens on single slides cut at a thickness of $2 \mu \mathrm{m}$. A-B. Stained with Regaud's iron haematoxylin. C. Stained with methylene blue-azurII. 
croscopy (= LSCM), micro-CT scanning $(=\mu \mathrm{CT})$, scanning electron microscopy and others. However, a drastic change occurred a few years ago, triggered by the appearance of highly sophisticated tools for preparing computer 3D visualizations for morphological research, available at relatively low expense.

Already since the 1970s, attempts were carried out to make use of the computer for morphological reconstruction and visualization (see Huijsmans et al. 1986 for review). These early approaches were throughout contour based; the contours of structures to be visualized were displayed in correct positions relative to each other. To name only one of those, relatively popular was $P C-3 D$ in the $1990 \mathrm{~s}$ (e.g., Page 1998). Other (later) approaches (e.g., NIH Image-similar to the currently still popular Image/J) included the displaying of structures as areas of sections similar to contour visualization (e.g., Bohn \& Heinzeller 1999). The actual breakthrough with regard to "physical" (in contrast to "virtual" sections like those obtained from $(\mu-)$ CT, LSCM or other non invasive method) serial section reconstruction came with the ability of creating structure surfaces. Such surface rendering has been applied for morphological purposes for at least twenty years. However, until recently such applications, due to the elaborate computer resources required, were restricted to platforms like Unix or Silicon Graphics workstations (e.g., Haas \& Fischer 1997; Sätzler 2001), which were not easily accessible for most workers. Since a few years ago, personal computers (including the Macintosh platform) have reached a performance level allowing routinely applying reconstruction with surface rendering visualization.

In particular, the software system $A M I R A^{\circledR}$ (Mercury Computer Systems Inc., Chelmsford, MA USA; Appendix 1.6. Specifications of software), which will be treated in detail in the present methodological compendium, has attracted increasing attention and has been used for micromolluscs successfully already (e.g., Neusser et al. 2006; Ruthensteiner 2006). This in turn drew general interest on the ribbon-forming resin sectioning method, which is a most useful prerequisite for computer reconstruction. AMIRA is an extensive software package with application fields reaching far beyond light microscopical section reconstruction (Brandt et al. 2005; Stalling et al. 2005). Manuals of this software cannot cover all specific details of "physical" section treatment, because for this application alone among a broad range of strategies for most processing steps must be chosen. Respective methodological information, however, frequently is omitted in result-based papers (e.g., Neusser et al. 2006). Accordingly, a "manual" explaining the crucial procedures is overdue. It can serve as an aid for both carrying out respective procedures for students and interpreting result for readers. Since my practical experience in computer reconstruction/visualization mainly concerns $A M I R A$, details in the following refer to this system. Nevertheless, many of the processing steps and strategies will be transferable if working with alternative systems.

For all these reasons it seems appropriate to include instructions for both the histological and the graphical procedures. It is aimed to provide a practical guide covering all processing steps, beginning with anesthetization of animals, which play a role for the final goal, the preparation of 3D models to be utilized for analysis and visualization. This may appear to be an overambitious goal. However, to limit the total extent, only such information is provided that seems particularly significant, or which is not available elsewhere. Otherwise, sources for additional information are quoted. The information provided is based on years of practical experience in laboratory procedures and graphical reconstruction methods, with a focus on AMIRA in recent years, by the author. It is intended to pass on this experience to the scientific community, with the particular aim to increase research efforts on micro- 
molluscan morphology. Hence, the present paper may be regarded as a continuation of Geiger et al. (2007), by substantially extending a topic that has been treated there briefly already.

It remains to be mentioned that many of the methodological details given are mainly/only based on uncontrolled observations that led to personal "philosophies". Accordingly, there is plenty of room for anyone to further optimize procedures. This not least regards the array presented for reconstruction with $A M I R A$. Since only a small fraction of the whole functionality of this software package was applied, there may have been a number of additional aids for facilitating the procedure overlooked.

\section{Methods}

\section{Morphological-histological treatment}

\subsection{Specimen treatment}

1.1.1 Relaxation. Though not absolutely necessary, it is very helpful for further treatment to relax specimens prior to fixation. This is more important in shell-bearing molluscs than in species without shells and particularly important in gastropods with coiled shells. The main purpose is to avoid the soft body retracting into the shell. Instead, it should exhibit an extended condition in approximation to life appearance, which is helpful for both examination and final visualization. There is some information in the literature available on that matter (e.g., Smaldon \& Lee 1979; Geiger et al. 2007), where usually different requirements in accordance to environment (marine-freshwater-terrestrial) are outlined.

At this point, the usefulness of magnesium chloride $\left(\mathrm{MgCl}_{2}\right)$ should be emphasized one more time. If applied in a suitable way, it works well for marine and - this may be less well known-freshwater animals. With regard to the former case, a persistent error on the concentration extending from older (e.g., Adam \& Czihak 1964) to recent (e.g., Geiger et al. 2007) literature specifications must be pointed out: The concentration of 7 or $7.5 \%$ given to be isotonic with seawater is at least to say ambiguous. In a strict chemical sense, it is half this concentration-about 3.5\% that is isotonic (3.57\% solution: $3.57 \mathrm{~g}$ anhydrous $\mathrm{MgCl}_{2}$ or $7.62 \mathrm{~g} \mathrm{MgCl}_{2} \cdot 6 \mathrm{H}_{2} \mathrm{O}$ filled with distilled water to $100 \mathrm{ml}$ ). This concentration definitely provides much better results at relaxation and subsequent fixation than the 7 or $7.5 \%$ anhydrous $\mathrm{MgCl}_{2}$ solution. For freshwater animals, where relaxation generally is more difficult than in marine animals, anhydrous $\mathrm{MgCl}_{2}$ will provide good results at much lower concentration, ranging from $0.5-1 \%$.

To achieve satisfactory relaxation, further factors like the type of solution applied play a role. No less important are duration and temperature, which can be varied in combination with changing concentrations (proportion of $3.5 \%$ anhydrous $\mathrm{MgCl}_{2}$ : sea water). The duration usually ranges between 5 and 30 minutes if the full concentration is applied. Sometimes it is required to replace seawater only partly (e.g., 50\%) by the magnesium chloride solution to avoid immediate retracting at the beginning of the relaxation process. Cooling down to as low as $5^{\circ} \mathrm{C}$ during relaxation often proves to be helpful.

1.1.2 Fixation. A wide range of fixatives can be used for the light microscopical purposes treated in the following. Recipes are available from many comprehensive methodological reviews (e.g., Böck 1989; Kiernan 1999; Geiger et al. 2007). Most commonly used are ethanol, formaldehyde — both at varying concentrations - or Bouin's fixative fluid. In contrast to older opinions (e.g., Adam \& Czihak 
1964; Böck 1989), ethanol is not listed as an appropriate fixative for histology in recent methodological accounts (e.g., Geiger et al. 2007). It should be pointed out that ethanol (approximately 75\%), nevertheless, may lead to useful histological conditions. Because of several advantages, like low toxicity, ease of application during collection in the field or the potential of simultaneous utilization of specimens for molecular purposes, it may serve as useful compromise to be used for histology, too. This implies that older material stored and possibly also fixed in ethanol may be used for detailed histological analyses (e.g., Haszprunar 1988). Histological quality of such material varies a lot and seems to be due to carefulness of the original fixation procedure, duration of storage or quality and kind of the storage fluid with regard to concentration of ethanol and denaturing substances. In general, the impact of the kind of fixative solution on the tissue quality is overestimated. No less important are criteria like post-fixation treatment including exchange of substances, temperature and $\mathrm{pH}$ buffering during storage etc. Discussing such details would go beyond the scope of the present paper.

Another method of fixation to be mentioned is that used for TEM (see Appendix 1.1. TEM fixation). The central benefits of such a relatively complex treatment, consisting of a two step fixation by complicated and hazardous solutions, are that tissue preservation is nearly optimal and resin embedded specimens may be used for both light microscopy and TEM (and also for scanning electron microscopy if critically point dried instead of embedded). However, there are also disadvantages: If investigated by light microscopy, the overall appearance of tissues is very homogeneous providing low contrast differences compared to conventional light microscopical fixations. This may lead to difficulties in telling tissue components apart. Because of minimizing shrinkages effects by TEM fixation, tissues appear to remain very dense without artificial gaps at the cellular level. This leads to another negative effect: infiltration of the embedding resin is more difficult, causing a lower limit to possible specimen size.

\subsection{Further histological procedures}

1.2.1 Shell (hard part) treatment. In micromolluscs it often is required to decalcify specimens after fixation. There are different ways to do so. The selection mainly depends on previous fixation and storage of specimens (see Appendix 1.2. Decalcification for examples). If possible-for larger specimens - after decalcification, the remaining non-calcareous shell components, such as periostracum, should be removed with the help of a needle or forceps. This facilitates exchange and penetration of substances during the embedding process and also to prevent disturbances during sectioning. Similarly, the operculum should be removed in some gastropods.

1.2.2 Embedding. Of the various ways of embedding for histological sectioning (see e.g., Adam \& Czihak 1964; Böck 1989; Glauert \& Lewis 1998), embedding in epoxy resins is the recommended choice for micromolluscs. Spurr's low viscosity resin, Araldite and Epon (see Appendix 1.3. Resins and 1.4. Embedding procedure for protocols) provided satisfying results. This way of embedding and sectioning is to be preferred to paraffin (paraplast) sectioning because sections can be prepared much thinner and show much less distortions. Individual components do not become shifted relative to each other, because the embedding medium is not removed during staining. The advantage of epoxy resins over hydroxyethyl-methacrylates (e.g., Technovit 7200 VLC, Heraeus Kulzer GmbH, Wehrheim/Ts., Germany), where the embedding medium is also retained in sections during staining, is their non-hygroscopic nature. This allows the "wet sectioning" technique, where sections are floating on a water 
trough attached to the knife, a prerequisite for the ribbon formation technique treated in detail in the following. Furthermore, in contrast to hydroxyethyl-methacrylates, open flat embedding moulds can be used for epoxy resins, substantially facilitating orientation of specimens prior to polymerization.

There are also drawbacks of epoxy resins, compared to the embedding media mentioned above: (1) Such resin causes changes to histochemical properties of tissues (Böck 1984). This substantially limits the options for applying histochemical and multiple stains. For example, basophil-eosinophil components characterized by different $\mathrm{pH}$ values can hardly be discerned in expoxy resin. Thus, for routine procedures mostly only single stains are used. However, as can be judged from experience and comparison with multiple stains like "Azan" and "HE" (see e.g., Böck 1989 for protocols), this is a minor problem since final identification of structures is nearly always based on differences in brightness and contrast, rather than on colouration properties. Particularly for the 3D software dealt with below this is not a problem because labelling of structures is done on grey scale images. (2) They are more hazardous than other embedding media (see Glauert \& Lewis 1998 for specifications), a problem that particularly concerns Spurr's resin. This does not only concern the resin component, but also one transitional solvent, propylene oxide. This carcinogenic chemical is often used and recommended as the standard transitional solvent in most methodological books (e.g., Böck 1989; Bozzola \& Russell 1998; Glauert \& Lewis 1998) to follow ethanol dehydration and dilute the resin for infiltration. However, as already known from older protocols (e.g., Adam \& Czihak 1964), this works just as well with acetone, which is hardly toxic at all (see Appendix 1.4. Embedding procedure). (3) Penetration of tissue with epoxy resins is relatively difficult. Therefore, the viscosity of the resin used is important with regard to the properties like kind of fixation and specimen size. Glauert \& Lewis (1998) provide detailed information on viscosity of different resins. (4) Another shortcoming of epoxy resins is their hardness. The strain put on the knife at sectioning is relatively strong and increases with block width. Because of pts. (3) and (4) the specimen size is limited - normally to a maximum diameter of $3 \mathrm{~mm}$.

If comparing the resins we employ, Spurr's resin and Epon are more viscous than Araldite and, therefore, are to be preferred to Araldite for specimens treated with TEM fixation, where penetration is more difficult than in conventionally fixed material (see also 1.1.2). In contrast, Araldite offers the best sectioning properties, as it least tends to show scratches. This may be due its relatively high elasticity. In addition, it polymerizes more reliable than, for example, Spurr's resin.

For flat embedding, moulds of silicone rubber or polyethylene with a trough size of about 5-10 mm $\times 12-17 \mathrm{~mm} \times 3-5 \mathrm{~mm}$ proved to be efficient. The polyethylene BEEM ${ }^{\circledR}$ P.E. Flat Embedding Mould (Ted Pella, Inc., Redding, CA, USA) can be warmly recommended for small specimens (see Fig. 2A for a block resulting from such a mould). This kind of mould is much cheaper and longer lasting than silicone rubber moulds. After transfer of specimens to the moulds, with the help of a needle or toothpick, they should be moved to the mould bottom and orientated to a position most suitable for sectioning. Prior to polymerization, moulds with resin plus specimens can be exposed to partial vacuum to facilitate penetration of the resin into the tissue. After this process and after the beginning of warming for polymerization, orientation could be controlled one more time and corrected if required. If specimen orientation is not as desired after polymerization, specimens can be trimmed out of the block together with some surrounding resin and re-embedded in (the same) resin in a corrected position.

For purposes such as controlling alignment (see 2.6) of sections, it is helpful to have images of total embedded specimens inside the polymerized block. When taking such images with the com- 
pound microscope, orientation of the block with the bottom towards the objective is recommended particularly for small specimens (the high magnification objectives required have little working distance). The block's bottom, like all sides of the block formed by the mould, is somewhat rough and opaque. It can be made perfectly clear by applying a drop of immersion oil and covering this with a coverslip (Fig. 2B).

1.2.3 Sectioning. For general procedures dealing with resin sectioning see Reid and Beesley (1991) and Bozzola and Russell (1991). Although both books are primarily dealing with TEM sectioning, they provide basic information on material and gear to be used for resin sectioning and also treat light microscopy specifically.

1.2.3.1 Trimming. To achieve ribbon formation of "semithin" sections, the block should be specially trimmed. Trimming should be carried out with a single-edge steel blade (preparatory trimming) and/or razor blade (final trimming) under the stereo microscope. The following description refers to a block shape resulting from typical flat embedding moulds (Fig. 3). The block is rotated $90^{\circ}$ with respect to the mould/polymerization orientation, Figs 3A, 4A), so that upper and bottom face are directed to the original sides of the block. This is advantageous because of resin consistency (often more brittle at the block surfaces than inside the block) and spatial reasons (the specimen often lies very close to the block's bottom). Both the upper and the lower face are trimmed to an angle of about $30^{\circ}$ (Fig. 3B) (with regard to the longitudinal axis of the block) and in parallel to the sides of the block-initially not too close to the specimen. Since the specimen should have been placed close to the bottom of block, it comes to lie at one side of the resulting cutting face, if viewed from the anterior end of the block (Fig. 3A,D-F). It is recommended to perform final trimming from the sides and application of the contact cement (see below) after some sections were taken off with the microtome to achieve a clear and absolutely plain cutting face (Fig. 3D,E).
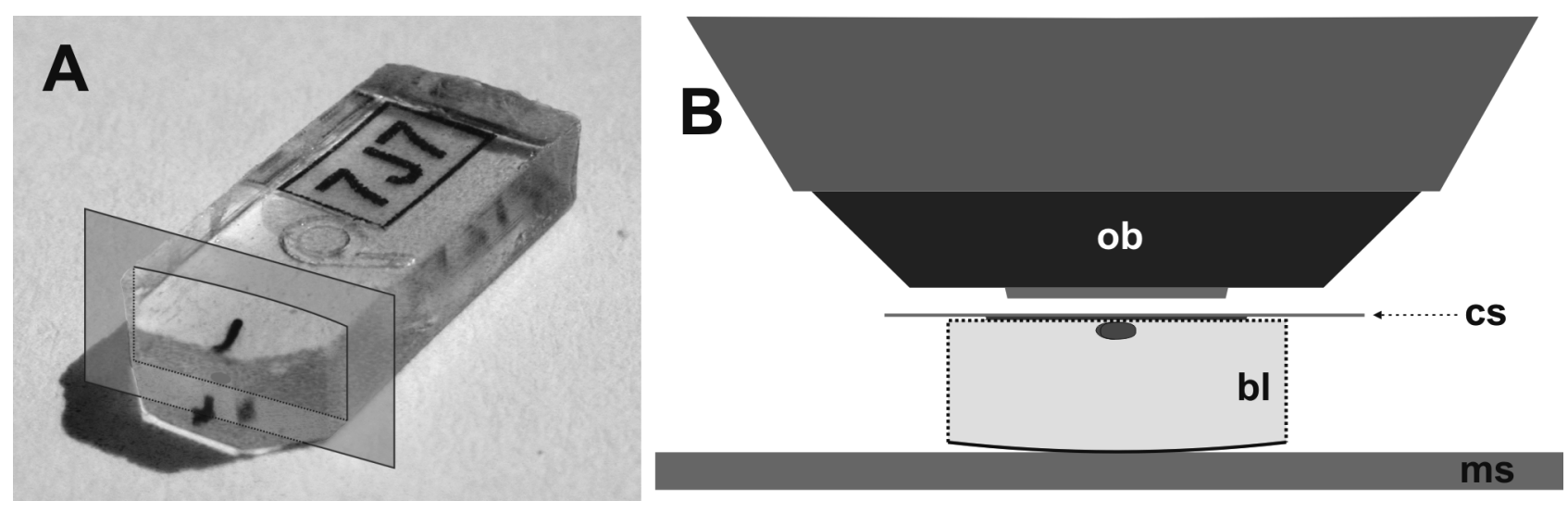

Figure 2. Resin block with embedded specimen and photographing of specimen on the compound microscope. Specimen is small solenogaster (glutaraldehyde-osmium tetroxide fixation, Spurr's resin embedding, same block and specimen as in Figs 3, 4 and 11, see Fig. 11 for photographic results). A. Total (untrimmed) block containing a label (transparent paper, inkjet printer) embedded together with the specimen. Blue transparent oblique rectangle indicates the "optical plane" of the block figured in B. B. Arrangement for taking specimen images. Block upside down relative to A. bl, block; cs, coverslip (on immersion oil); ms, micro slide; ob, microscope objective. 
For final trimming - after initial sectioning - a polarizing system at a stereo microscope is very helpful. This allows adjustment to have the (cutting) face either transparent (looking inside the block to see the specimen) or reflective (to see the surface; Fig. 3E,F). Scratches from the microtome knife on the cutting face (Fig. 3E) are a useful reference for adjusting angles at final trimming. The cutting face should be trimmed to a rectangle (Figs 3A,E,F, 4A). This is better than trimming to a trapezoid shape with a longer lower edge, as suggested by e.g., Blumer et al. (2002), because the resulting protruding corners of sections disturb the alignment of ribbons on the slide (see below). Also, it is more economical in terms of wear of knife and there is simply no advantage for trapezoid trimming. The dimensions of the rectangle follow the dimensions of the specimen to be cut (height and possibly width - referring to the orientation at sectioning as given above; Figs 3A, 4A) and the minimum width of $1 \mathrm{~mm}$. So, for specimens with a height less than $1 \mathrm{~mm}$, the rectangle is wider than high. Very small specimens typically come to lie on one side (the original bottom-Fig. 2) of the block, with some empty resin on the other side. The minimum of $1 \mathrm{~mm}$ width is recommended because the upper and lower edges form the attachments between sections at ribbon formation. The longer they are, the more stable is the connection between the sections and, thus, of the ribbon. There is also a maximum width of about $3 \mathrm{~mm}$, because of the strain put on the knife at sectioning (see also problems of epoxy resins 1.2.2; Reid \& Beesley 1991).

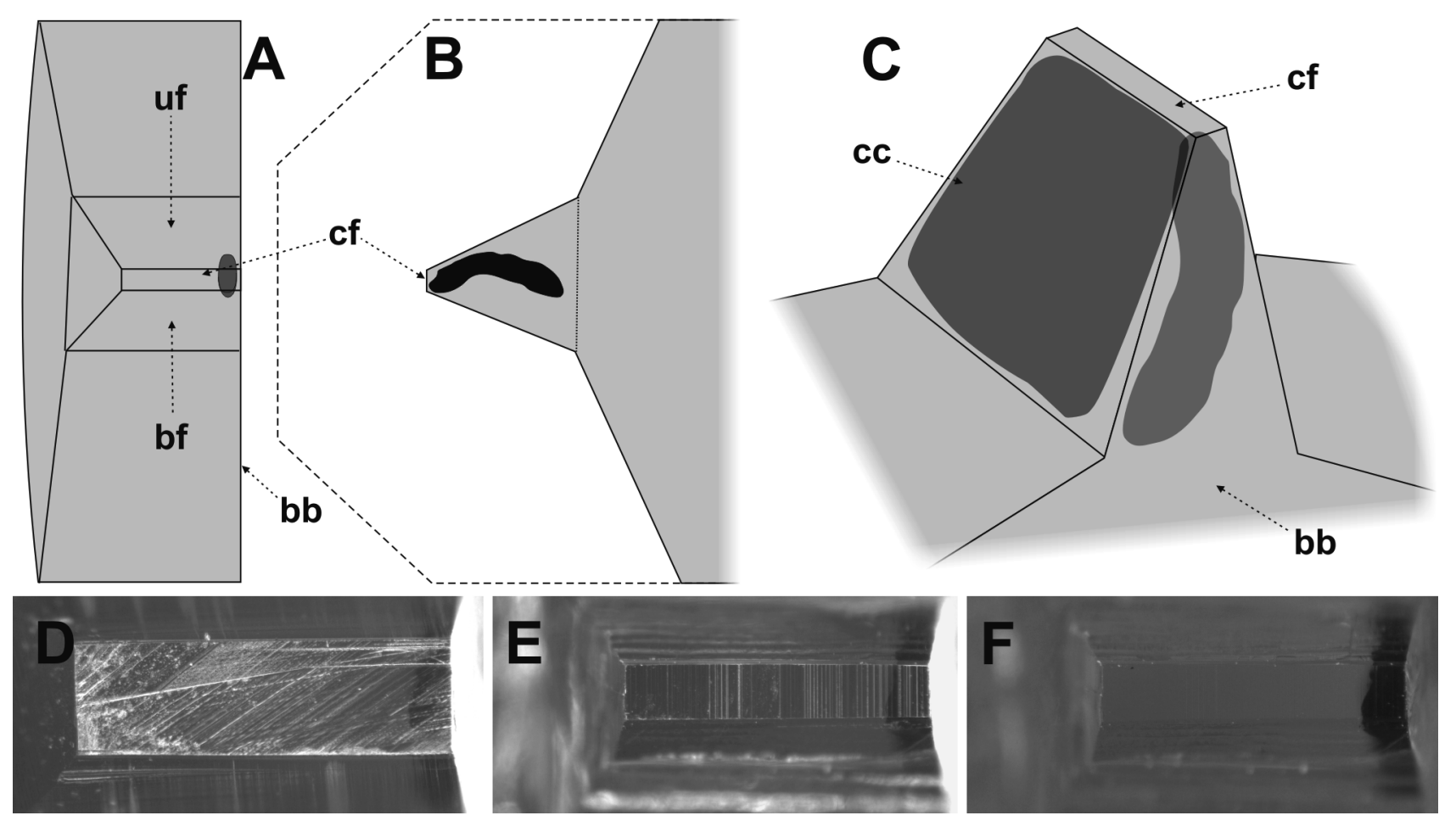

Figure 3. Trimming of the block and placement of the contact cement. A-C. Schematic drawings showing final trimming shape from different angles. Specimen (blackish/greyish) is small solenogaster (same as in Figs 2, 4 and 11). A. View onto the cutting face side of the block. B. View onto the original (mould's) block's bottom. Stippled line gives the original contour of the block prior to trimming. C. Oblique view showing the position of the contact cement (reddish transparent). D-F. Cutting face photographed at identical magnification on a stereo microscope. D. Final trimming for cutting face only, prior to initial sectioning. E. Final trimming after initial sectioning. Face shows scratches from Ralph knife. F. Same as E. but with polarizing filter. cc, contact cement; bb, block's bottom; bf, bottom face; cf, cutting face; uf, upper face. 
1.2.3.1 Contact cement application. As the final step before going into sectioning, the adhesive is applied. For this purpose the contact cement Pattex Compact (Henkel KGaA, Düsseldorf, Germany) is used. The American brand Weldwood Contact Cement (DAP Products Inc., Baltimore, USA) was also tested and provided similar results. The latter cement was used in the protocol by Henry (1977), which represents the basis of the present method. Henry assumed that any contact cement with similar properties would work as well for that purpose. The cement usually - it ages in the tube and becomes more viscous - needs to be diluted with, for example, xylene or toluene. This can be done by separating a small fraction in a small vessel like an Eppendorf tube, where the solvent (few drops) can be added. The consistency should be such that it is not thready; too much dilution, however, can soften the resin underneath the cement. The cement is applied to the underside of the block, the surface, which is first approached by the knife (Figs 3C, 4). This is done by transferring a small drop with the tip of a needle under the stereomicroscope to the block, where it is spread with the needle tip. The cement layer should be kept thin. It must be avoided to get cement over the edges of the surface on the sides of the block and on the cutting face. In the first case it would pull strings at the side of sections and attach wrong portions of sections to each other. In the latter case the knife could be damaged at the beginning of sectioning. For those reasons, one should leave a little space to the side edges but bring it as close as possible to the cutting face, so that ribbon formation will work immediately or soon after the beginning of sectioning. During the application, the cement may remain liquid for about 15 seconds - the time available for this process. It is possible to remove the cement completely with the tip of a needle. This may be required if the application did not work as desired or if sectioning is interrupted for longer than one to two days so that the cement loses its adhesive property.

1.2.3.2 Knives. For sectioning, either Ralph glass knives (Bennett et al. 1976) or a Histo Jumbo diamond knife of Diatome AG (Biel, Switzerland; Blumer et al. 2002) can be used. A Ralph glass
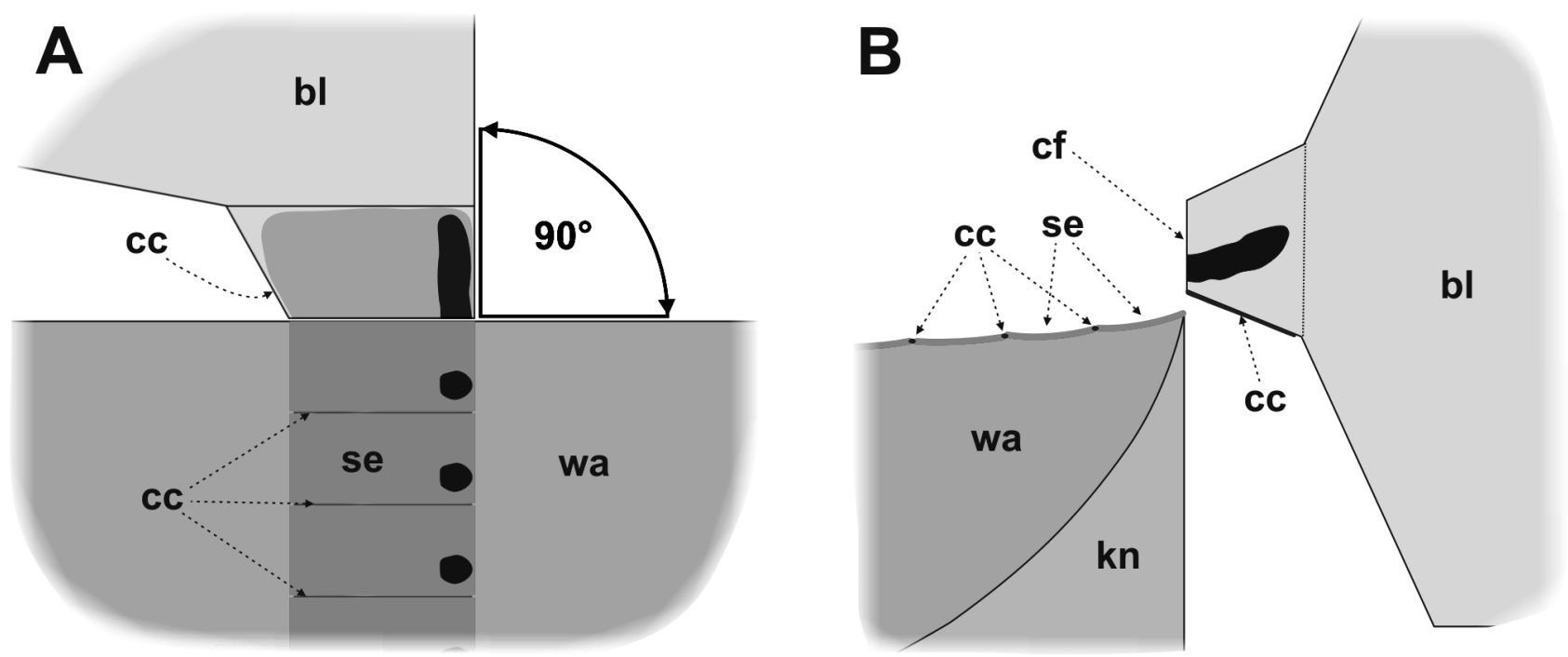

Figure 4. Schematic drawings of ribbon formation during sectioning. Specimen resembles the small solenogaster of Figs 2, 3 and 11. A. View from above onto water surface. An angle of $90^{\circ}$ of the block (original block's/mould's bottom) towards the knife is useful for alignment during 3D reconstruction (compare to Fig. 11). B. View from the side with "longitudinal section" of sections, water in trough and knife. cc, contact cement; cf, cutting face; bl, block; kn, knife; se, sections; wa, water. 
knife can be prepared with special breaking tools of different manufacturers (e.g., Leica Histoknifemaker, Leica Microsystems GmbH, Wetzlar, Germany; Pelco Ralph Glass Knife Maker, Ted Pella, Inc., Redding, CA, USA) by breaking rectangular portions of $25 \mathrm{~mm}$ wide glass strips. We only have experience with the Leica Histoknifemaker, which unfortunately is no longer available for sale. For good Ralph knives the utilizable part in the middle of the knife's edge has a length of about $15 \mathrm{~mm}$. A horizontal trough must be attached to the knife (Fig. 5A); inner dimensions may be: $25 \mathrm{~mm}$ width, $80 \mathrm{~mm}$ length, $9 \mathrm{~mm}$ depth) usually made from plastic. If filled with water this must reach the cutting edge of the knife. The trough is sealed with dental wax towards the knife to make it watertight. For Ralph knives, the lowest possible sectioning thickness is $1.5 \mu \mathrm{m}$.

The Histo Jumbo diamond knife is provided with a large water trough by the manufacturer. Knives of this type are available at cutting edge lengths of 4, 6 and $8 \mathrm{~mm}$. Section thickness as low as $0.5 \mu \mathrm{m}$ is not a problem with such a knife. It has advantages over the Ralph knife, as it produces sections of constant thickness without losses and, thus, it is more reliable at forming the ribbons. Also, it produces fewer sectioning artifacts like scratches and scatters (Fig. 6E,F). Other disadvantages of Ralph knives are that they are very difficult to prepare and that knife position becomes worn during the sectioning process so that position needs to be changed regularly. Disadvantages of the Histo Jumbo diamond knife are the lower sectioning speed (according to manufacturer's specifications) and the risk of destruction of the knife. If hard parts like the radula or operculum bear components problematic for sectioning or sand grains are attached to the specimen, this can lead to the loss of (part of) the knife. In addition, with about $50 \mathrm{~mm}$ only, the trough of the Histo Jumbo diamond knife is too short for long ribbons. For all these reasons we keep using both knife types in our laboratory.

1.2.3.3 Sectioning procedure. A microtome is required allowing low advance steps (starting from $0.5-2 \mu \mathrm{m}$ ), which is provided with specific lightning units and a mechanism providing that the specimen does not get in contact with the knife, by either "retraction" or side movement, during the return stroke. These prerequisites are principally present in all microtomes dedicated to TEM sectioning
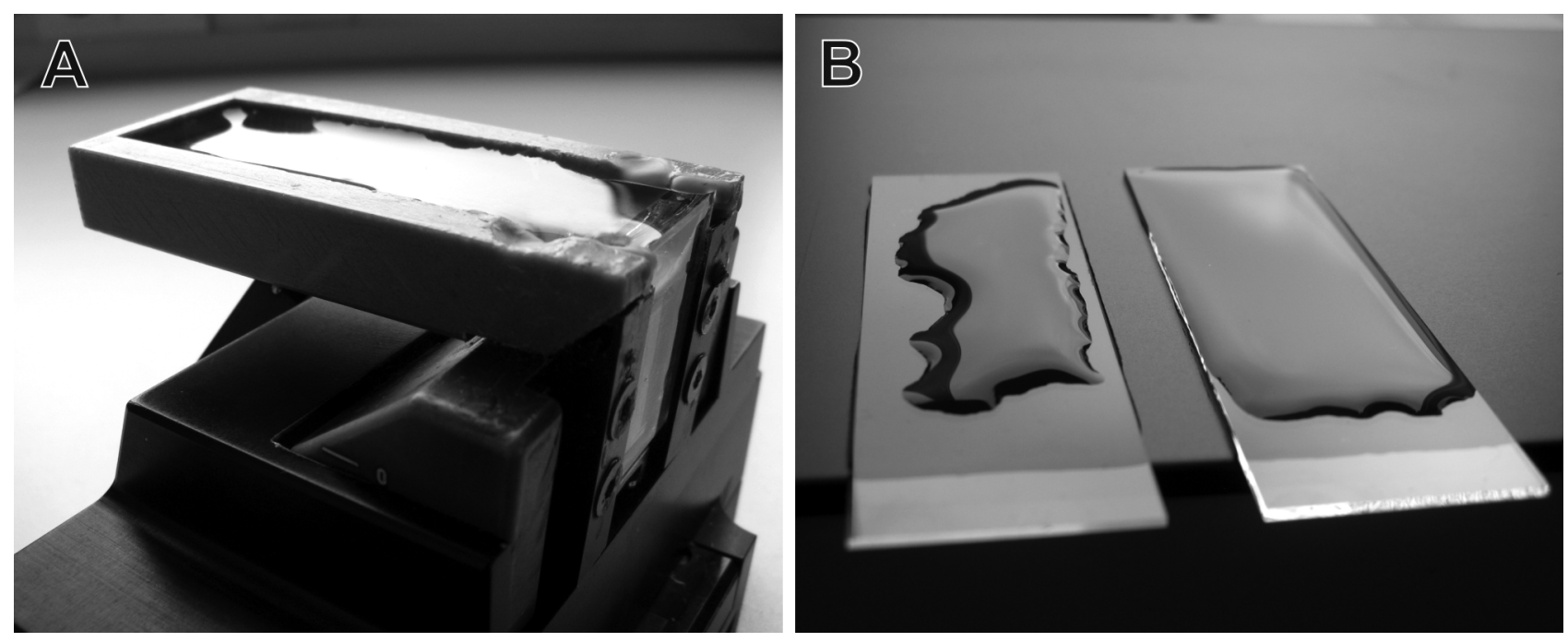

Figure 5. A. Ralph knife fixed to the Microm HM 360 knife holder with a self prepared trough. The latter is sealed with dental wax towards the knife and filled with water. B. Demonstration of suitability of micro slides for taking up section ribbons. Left slide is untreated and cannot be used because it is water-repellent. Right slide is suitable since water remains at the edges. 
("ultramicrotomes") but also in modern microtomes specifically optimized for "semithin" sectioning (e.g., Microm HM 360, Microm International SA, Lausanne, Switzerland; Leica RM2265, Leica Microsystems GmbH, Wetzlar, Germany). The latter are cheaper and may be preferred, as settings of cutting and return stroke speed may keep time expenditure lower.

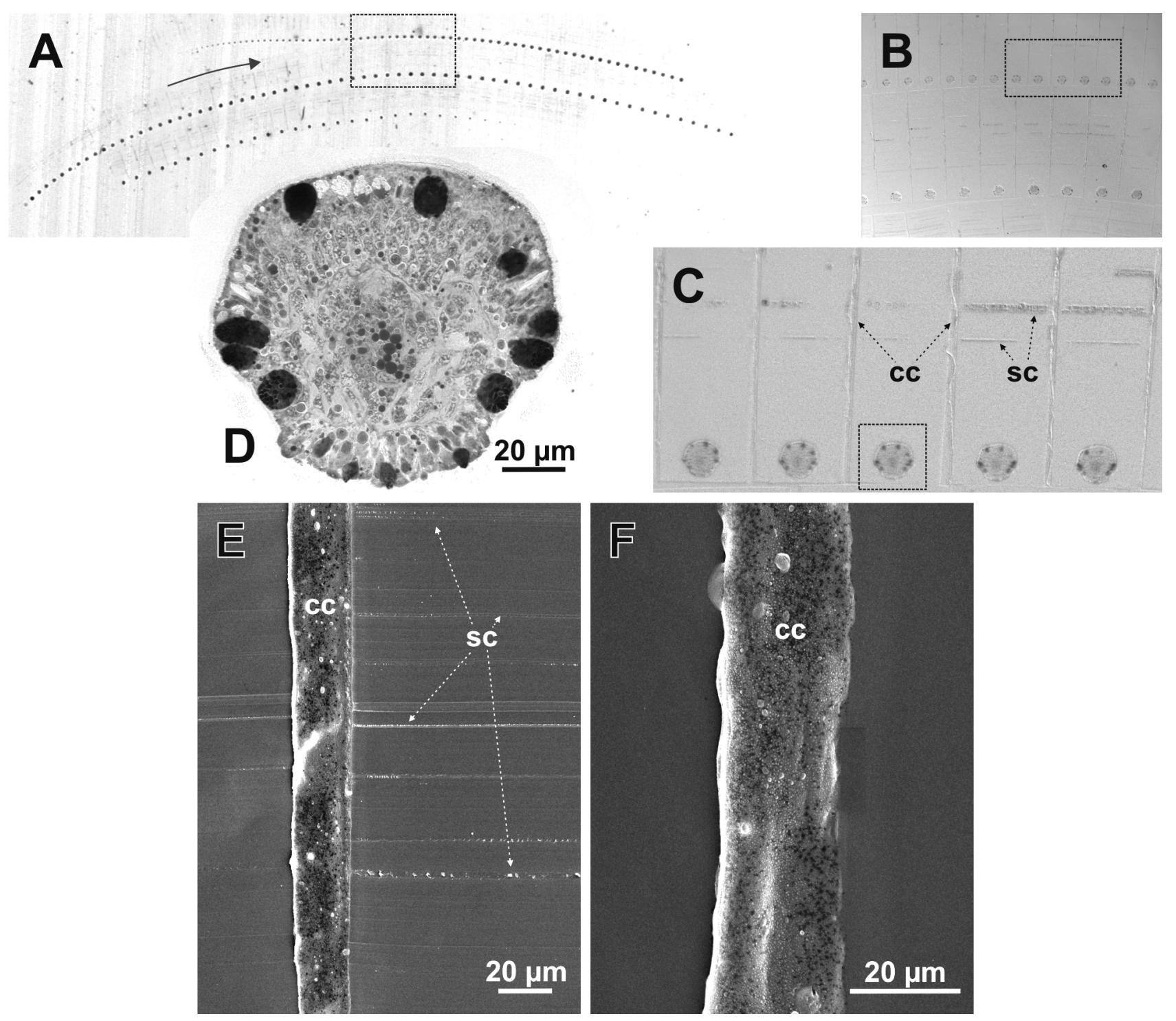

Figure 6. Results of section ribbon formation. A-D. Series on a single slide of a Chiton olivaceus larva (cross sectioned, $1.5 \mu \mathrm{m}$ section thickness, glutaraldehyde-osmium tetroxide fixation, Spurr's resin embedding, methylene blue-azurII dye) at increasing magnification. A. Total series scanned on a flatbed scanner at transmission lighting. Red arrow gives the "read direction". Stippled rectangle is enlarged in B. B-C. Details of the series showing the arrangement of sections in certain areas photographed on a stereo microscope. Stippled rectangle of B. is enlarged in C. Stippled rectangle of C. is enlarged in D. D. Single section (specimen part-in the region of the prototroch) of the series photographed at a compound microscope with slight differential contrast applied. E-F. Scanning electron micrographs of details of a section ribbon (1.5 $\mu \mathrm{m}$ section thickness, Araldite embedding) showing the adhesion region between two sections to demonstrate that the contact cement remains in the region of the sections. E. Ribbon sectioned with a Ralph knife. Sections show slight scratches-too small to be visible at the mounting medium covered sections on a compound microscope. F. Ribbon sectioned with a Histo Jumbo diamond knife. Sections show perfectly smooth surface. cc, contact cement; sc, scratches. 
The sectioning procedure is fairly similar to ultrathin sectioning. Sections are floated on the water surface of the trough, whereby the last one produced remains attached to the knife's edge (Fig. 3). The newly produced section shifts the one produced before away from the knife's edge towards the trough. Sections remain attached to each other and in this way form a ribbon (Fig. 4). When the latter has reached a length of about 40-50 mm maximum (up to 100 sections, somewhat shorter when using the Histo Jumbo diamond knife) it should be detached. It is recommended do detach it a few sections behind (loosening the connection between two sections) instead of directly from the knife's edge. By doing so, fewer disturbances are caused to the whole process. The sectioning process, usually carried out by the motor of the microtome, does not necessarily have to be interrupted for this purpose. Ribbons usually are quite stable and can be manipulated with the help of preparation needles. Detached ribbons are gathered in the order of their planned arrangement on the slide in the posterior part of the trough. If Ralph knives are used, the position of the knife must be changed when the cutting edge part is spent and produces sectioning artifacts like scratches. Nevertheless, a number of ribbons and several hundred sections may be produced with a good knife in good operating conditions. If the block has been trimmed in a way that the specimen is on one side and has empty resin on the other side (see above, Figs 4A, 6C), the knife must be shifted only for the distance of the specimen width. Usually the empty resin can be sectioned with a worn knife's edge.

The procedure given herein is based on the idea presented by Henry (1977). In contrast, however, Henry applied contact cement to the upper block face too (Fig. 7A). When tested, this showed that the formation of the ribbon initially worked well, but failed after a while and resulted in the destruction of the ribbons. Subsequent close examination of the block revealed that the - in dry condition — rubberlike cement on the upper block face remained uncut and protruded over the cutting face because of the block is shortened by sectioning (Fig. 7B). This appears to result from the missing counter pressure, which is applied to the cement layer on the bottom face due to the adjacent block resin. As soon
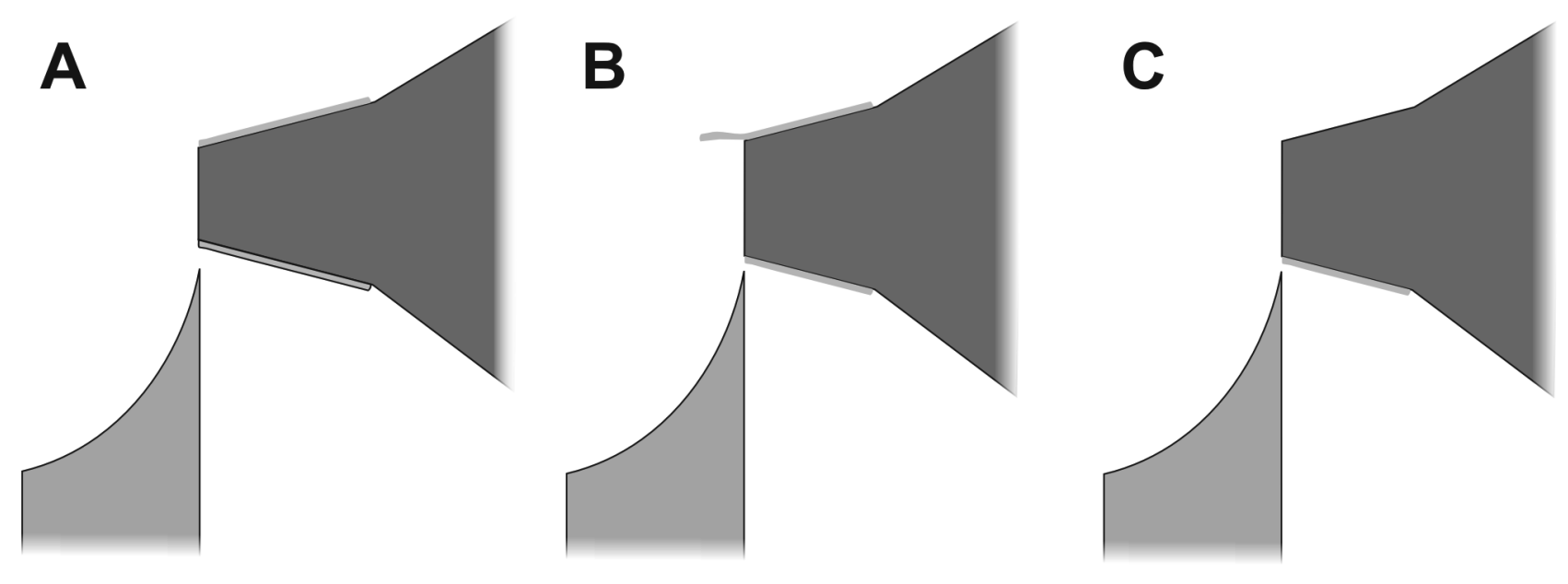

Figure 7. Comparison of the method given by Henry (1977) and the one proposed herein to achieve formation of ribbon at sectioning by application of contact cement. A. Uncut block with cement on both the bottom and the upper face as suggested by Henry. B. Resulting condition after taking off some sections when cement is applied as in A. C. Cement applied to the bottom face only is sufficient for reliable ribbon formation. Red, contact cement; yellow, block; blue, (Ralph) knife. 
as the upper freely-protruding cement has reached a certain length, it will catch the sections close to the knife's edge and in this way destroy the ribbon. Simply omitting the upper cement layer (Fig. 7C) immediately provided good results and reliable ribbon formation. It should be noted that there were further approaches to achieve ribbon formation with resin sections. Royer (1988, quoted in Reid \& Beesley 1991 and Bozzola \& Russell 1991) used a scoop prepared from laboratory plastic tubing to retain the untreated sections, attached to each other by coating the block with partially polymerized resin, forming the shape of a ribbon when transferring them to the slide. However, since the treatment of the block with this method seems quite complicated and the scoop allows maximal lengths of $25 \mathrm{~mm}$ for the ribbon, the method presented herein seems much more advantageous.

1.2.3.4 Transferring and mounting the ribbons. As soon as there is a sufficient amount of ribbons (up to 15) for a single slide in the trough, they need to be transferred to a micro slide. This represents one of the most difficult steps during the sectioning procedure. Initially the ribbons are aligned in the trough similar to their planned future arrangement on the slide. To keep disruption during the following processes low, it is useful to place the ribbons adjacent to each other; they tend to remain like this because of mutual attraction.

Another very important prerequisite is the pre-treatment of micro slides: They must not be waterrepellent, because this nearly always leads to various disturbances during ribbon mounting! Since nearly all commercially available slides bear this problem, they need to be specifically treated prior to use for the present purpose. A two step method to make them water-attracting has proven successful: At first, slides are immersed for at least a day in an ethanol (96\%) : ammonia (25\%) 9 : 1 solution. The second step is rigorously rubbing with a sponge and standard dish wash soap and careful rinsing in running tap water. When checked by holding the wet slides horizontally they should not exhibit any trace of water retraction from their edges (Fig. 5B). Until use they should be stored in distilled water upright in a cuvette with one end (about $15 \mathrm{~mm}$ in length) protruding out of the water.

When section ribbons are taken up from the water trough, a slide is taken by its dry end and slided from the posterior end of the trough underneath the section ribbons. With a needle, held in the other hand, ribbons are shifted towards the slide. If everything works fine, the ribbons - enforced by some unknown physical attraction - would move by themselves towards/upwards the slide. As soon as ribbons are safely positioned on the slide, the latter can be removed from the trough. The slide then is placed on a dark background for reordering the ribbons. Often it is necessary to carefully add more distilled water with a pipette to the floating ribbons on the slide prior to the stretching process. The latter is carried out on a $70^{\circ} \mathrm{C}$ hot plate. There the ribbons should be prevented from being attached to each other to allow individual stretching. Be careful not to touch the ribbons with any instrument while they are hot, since they would get stuck to this! When ribbons are cooled down, their arrangement on the slide is finalized. By cautiously draining the water around the ribbons, their final arrangement can be set prior to placing the slides in an incubator (about $60^{\circ} \mathrm{C}$ ). It seems important to note that all manipulations to floating sections and ribbons are best performed with preparation needles. Eyelash or bristle, such as recommended by Blumer et al. (2002), is unsuitable for most purposes, because they are too weak for most manipulations.

1.2.3.5 Staining and coverslip mounting. Ribbons, in general, adhere well to the slide, although once in a while sections will became detached during the staining procedure. It appears that heating 
slides with dry sections once for longer than 10 minutes on a $70^{\circ} \mathrm{C}$ hot plate is useful to prevent this. The risk for becoming detached is higher if sections bear more disturbances, like scratches.

Similarly, as for histological sections obtained with other methods, there are numerous distinctly differing staining protocols for epoxy resin sections in the literature (e.g., Böck 1984). I tested some of the more complex procedures, including removal of the resin with subsequent staining in cuvettes similar to paraffin sections. From the experience gained by this, I maintain that such extensive staining procedures are not worthwhile, because they provide no better results than simple staining by overlaying sections on the slide with a single staining solution (see also 1.2.2). Simple blue stains like toluidine blue or methylene blue-azur II (see Appendix 1.5. Section staining — for protocols) are perfectly suitable to depict relevant details at slightly varying blue tones and broad range of contrast and brightness nuances (Figs 6A, 8B).

For storage and easy accessibility of staining solutions, a syringe with attached filter is best suited. The filter prevents the contamination of sections with precipitations, which are continuously produced in such staining solutions. It is convenient to have an array of syringes with different concentrations of staining solutions available.
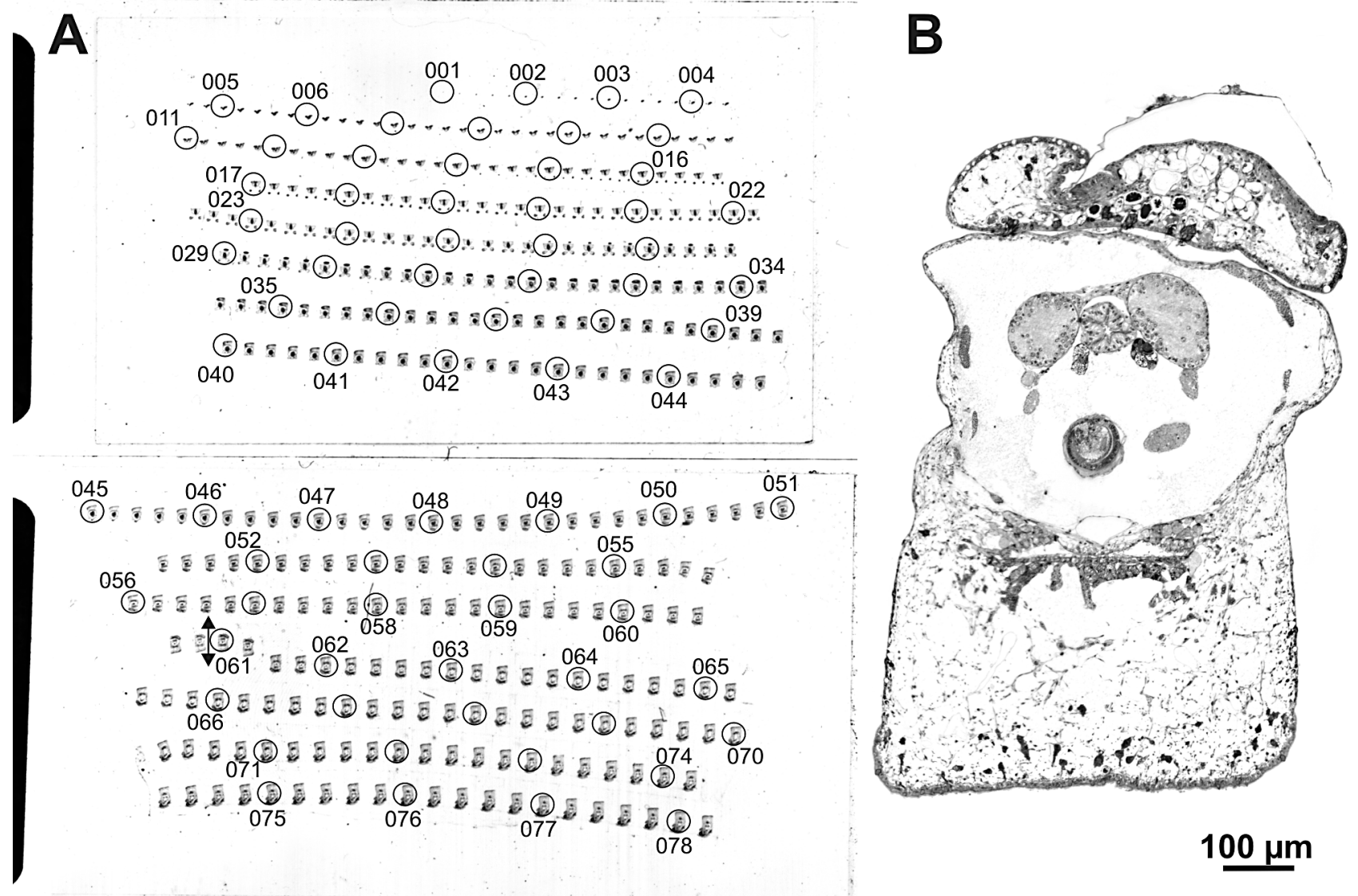

Figure 8. Sectioning chart of part of a series (A) and individual section (B) of the pulmonate Ovatella myosotis (juvenile, cross sectioned, in total about 1,800 sections, Bouin's fixative, Araldite embedding, $2 \mu \mathrm{m}$ thickness). A. Image scanned with a flatbed scanner at transmission lighting, circles and numbers added with vector graphics software (Corel Draw 11). Every fifth, a total of about 360, sections are utilized. B. Section nr. 059 (rotated $180^{\circ}$ ) in the region of the cerebral ganglia and radula pouch, photographed with a compound microscope with slight differential contrast applied. 
For staining, section(s) (ribbons) are covered with the staining solution on the horizontal micro slide. To accelerate staining, the slide is placed on a $60^{\circ} \mathrm{C}$ hot plate. Staining is discontinued by cautiously (no direct water jet onto the sections) rinsing the slide in running tap water and subsequently in distilled water. Slides are then dried by placing them on a hot plate and simultaneously drying excess water on top of the slide with filter paper.

The intensity of the staining process can easily be adjusted by altering the concentration of the staining solution and staining duration on the hot plate. Important factors influencing the staining intensity required are the kind of fixation used, kind of embedding resin and section thickness. To give an example, conventionally fixed material embedded in araldite requires much less intensity (e.g., stock methylene blue-azurII solution : distilled water 1:1, 20 seconds) (Fig. 8) than TEM-fixed material embedded in Spurr's resin (e.g., stock methylene blue-azur II solution full concentration, 60 seconds) (Fig. 6A). It is no problem to stain sections repeatedly in case of "understaining", but (partly) removal of a dye in case of "overstaining" with the help of, for example, ethanol, cannot be recommended. The resin of section and thus also the tissue becomes severely impaired thereby. It should be mentioned that there are "depth" limits for penetration of the dye into the section. Sections with 2 $\mu \mathrm{m}$ thickness do not get stained down to their deepest layer attached to the slide, which appears to be similar in different epoxy resins. This can be observed in tissue containing natural pigmentation.

For mounting slides with stained sections, regardless of the embedding medium used, araldite can be recommended. This is the same as applied for embedding. Carryovers from the latter purpose are appropriate. Araldite is suitable since it does not affect sections, like making them wizened or distorting them as occurs when using, for instance, Spurr's resin or even worse Eukitt (this is frequently used as a mounting medium for paraffin sections). Also, Araldite does not dissolve dyes out of the tissue.

During application of araldite for mounting, attention should be paid to the following: A surprisingly small amount is necessary for each slide. It spreads out rather slowly underneath the coverslip until it reaches the edges. Polymerization should take place at room temperature or at a maximum of $37^{\circ} \mathrm{C}$ (duration until full hardening is approximately three days), to avoid affects like those described above for other mounting media.

Experience on stability of dyes in combination with this mounting medium reaches back about 20 years. This shows that sections stained with dyes such as methylene blue-azur II do not exhibit noticeable fading.

\section{Analysis and graphical treatment}

2.1 General strategies and file sizes. As initial set up for the graphical analyses of a specimen, objectives like size and intended accuracy of analysis and visualization of a structure or specimen portion have do be determined. Autonomy of decision hereby is restricted by factors like expenditure of work involved, optical and digital resolution of images, as well as file size with regard to performance of hardware and software applied. In practice, it needs to be clarified (1) at which interval sections are used (each, every other, every fourth, etc.); (2) which optical magnification is used (objective, zoom); (3) which digital resolution is used for taking the images; (4) is the whole specimen or only (which) part of it processed?

Most of the following concerns - as would be mostly the case in micromolluscs - are analysis and visualizing of total specimens. The software introduced here, AMIRA (see Appendix 1.6. Specifica- 
tions of software and hardware applied), in its basic configuration allows using file sizes for the initial image stack file of up to $500 \mathrm{MB}$ (This and further file size specifications refer to a typical current PC array, such as the one given in Appendix 1, 6. Continuous increase of such dimensions is to be expected with further development of hard and software.). Since the system at this file size becomes unstable and slow, it is recommended to use file sizes not substantially exceeding $400 \mathrm{MB}$. If there are enough initial images available, which may not be the case in very small specimens with a small diameter and a small number of physical sections only (e.g., Nielsen et al. 2007, Fig. 15), it makes sense to chose a resolution for the image stack file at which the file size approximates $400 \mathrm{MB}$. This is advantageous since the segmentation process in general and surface rendering for delicate structures may be facilitated by high resolution. The size of an image stack file (Stacked Slice format —*.am, further on referred to as "image stack") can be calculated in advance by resolution and number of section images to be used. Pixels number of images $(\mathrm{X} \times \mathrm{Y})$ multiplied by the number of sections $(\mathrm{Z})$ results in voxel number. In the case of the 8-bit 256 grey level color format eventually used in AMIRA, voxel number is the same or slightly smaller than the byte number of the image stack file. This allows choosing the number of sections to be used for the image stack in advance. To give an example, if images resolution would be kept at 2 mega pixel (MP) throughout the process (e.g., 1,600 × 1,200), no more than 210 sections could be used. Image resolution may be reduced in favour of $Z$ resolution (number of section images) (see also 2.3). For treatment of general organization of a whole specimen, a number of 250-350 section images turned out to be suitable. Accordingly, at section thicknesses ranging between $0.5-2 \mu \mathrm{m}$ (or also 5-8 $\mu \mathrm{m}$ in paraffin series) and specimen sizes larger than $150 \mu \mathrm{m}$, one frequently cannot use every section for digital procession. Instead, equidistantly located sections are used. In such cases every other, every third or multiple of these are to be photographed. It can be highly recommended to either mark selected sections on the slide (with a permanent marker) or to prepare a sectioning chart (Fig. 8) as preparatory step. The latter way is to be preferred particularly for small specimens, since there is hardly any space on the slide available for marking the sections directly. To do so, slides are photographed or-better — scanned at high resolution, if possible with transmission lightning (Fig. 8A). By contrast enhancement usually even small sections can be made visible in the resulting digital image. Labelling and numbering of sections may be performed with standard vector graphics software. Sectioning charts as well as marked positions on slides are primarily useful as a directory for finding and identifying sections during the photographing procedure. They may be also useful later for finding individual sections during reconstruction works when it is required to return to the microscope.

2.2 Taking photographs of sections. Sections are photographed with a compound microscope equipped with a digital camera. Principles of microscopes and microscopic photography are treated in detail elsewhere (e.g., Rost \& Oldfield 2000). The microscope should be supplied with objectives suitable for specimen size. For minute specimens (smaller than $500 \mu \mathrm{m})$ a $100 \times$ or $60 \times(63 \times)$ objective is obligatory. Availability of a zooming option is very helpful. For taking images, the bright field or possibly weakly applied differential (= interference) contrast mode are suitable. For taking the digital images a dedicated microscope camera is to be favoured over a consumer camera, because of advantages in operation and optic quality. In contrast to (most) consumer cameras, microscope cameras can be remotely controlled from the computer. Parameters like focus or camera frame can be directly adjusted with the help of the computer screen, which substantially facilitates taking im- 
ages. Consumer cameras have optical lenses, which are useless for the microscope because the latter provides the optics by itself. Such lenses have to be compensated for by additional ones in the coupler unit. Though it can be imagined that such additional lens systems are not really of advantage for optic quality, such cameras provide reasonable images anyway. Digital resolution does not have to be very high. Usually $2 \mathrm{MP}$ or even less resolution is sufficient. Color mode plays no role, since the image format for digital procession is greyscale.

Before taking images, the most advantageous magnification is to be determined. To do so, the largest section or the one with the longest extensions relative to the typically rectangular shaped camera frame has to be located. Subsequently, by selection of the objective and if possible additional options, such as zooming, the magnification is adjusted in such a way that this sections just about fits into the camera frame. For alignment purposes it may be very useful to have the section edge next to the specimen (according to the embedding/trimming mode proposed here it is the block/mould bottom) photographed together with the specimen (see 2.6). If doing so, this has to be considered when determining the magnification for photography. The adjusted magnification must be retained for all images of the series. In the face of 3D procession, it is helpful, if orientation of all images is roughly the same when taking the images, which is most easy in case of ribboned sections. The image files have to be named (alpha) numerically in the order of their position in the series.

In case sections are missing at certain positions in the series, it is possible to use images of other sections for this position. If not every section is used, a neighbouring section to the lost one may be used. If every section of a series is supposed to be photographed, I also suggest using images of a neighbour section for one or even more missing sections. For alignment of the series at 3D procession (see below), it is required to have a section image on a "lost" position instead of an empty image and it also helpful for the segmentation procedure. However, these falsely positioned images should be recognizable as such during $3 \mathrm{D}$ procession.

2.3 Image pre-treatment. Images often need to be reduced in size prior to being loaded into AMIRA (X-resolution $\times$ Y-resolution $\times$ number of images should not exceed approximately $4.3 \times 10^{8}$ ). In addition, it is helpful to enhance images with regard to brightness, contrast or definition. To judge such image properties it seems useful to convert the color space already to greyscale (8-bit 256 grey levels) as used in AMIRA. All these adjustments can be carried out at once by a batch function with different standard image editing software systems. For this purpose, typically all mentioned adjustments are performed on a trial basis to a single section image. During this process respective adjustment settings are saved and subsequently applied to all section images of the series when prompting the batch function. Modified images are newly saved in standard file formats, like *.tif or *.bmp, which are suitable for $A M I R A$.

2.4 Software-general. AMIRA can be run on a broad range of operating systems, among those the recent major versions of Microsoft Windows and Macintosh System 10.x; it is fairly expensive (several thousand $€$ for the basic version), but affordable if there are specific contracts with scientific institutions. AMIRA is not the only tool for the present purposes. Aside of several commercially competing products (see e.g., Clendenon et al. 2006) there are more and more free software systems, with functions based on the same principals, becoming available. As highly promising for the present purposes appear 3D Slicer/FreeSurfer (Pieper et al. 2006; http://www.slicer.org/; http:// 
surfer.nmr.mgh.harvard.edu/) and Reconstruct (Fiala 2005; http://synapse-web.org/tools/reconstruct/ reconstruct.stm).

There is a detailed manual (User's Guide plus Reference's Guide, contents similar to that of the help function) for AMIRA, which has retained its general arrangement among the versions (2.3-4.1.1) used. However, experience with many users has shown that this as sole information resource is insufficient to guide through the applications treated in the following. The introductory tutorials are very clear and helpful for general understanding of procedures. Conversely, large parts of the further explanations appear to be dedicated to the advanced computer user and inappropriate for solving specific problems appearing during operation steps.

2.5 AMIRA-import - calibration. Finalized section images are loaded into AMIRA at once by selecting them all. The resulting file, the image stack, represents a 3D voxel image. For saving it, the *.am (amiraMesh binary) format proved to be most appropriate. As can be seen in the crop editor the default dimensions after import are set to 1 for $\mathrm{X}, \mathrm{Y}$ and $\mathrm{Z}$. This means that 1 voxel is cubic and one edge length represents one model unit and the specimen usually is compressed in length because the Z-value in reality is nearly always higher than the $\mathrm{X} / \mathrm{Y}$-values. Accordingly, the relative dimensions (X/Y vs. Z) have to be adjusted to provide an un-compressed or un-elongated overall shape of the specimen. In doing so, it makes sense to adjust the dimensions in a way that one unit matches a real dimension like mm or $\mu \mathrm{m}$. For micromolluscs the later dimension would be nearly always most suitable. This calibration can be done after data import or any time later prior to the generation of a LabelField file (see below). If this is performed after LabelField generation, identical dimensions like in the original image stack have to be inserted in the LabelField by hand in the crop editor. The $\mathrm{Z}$-value represents the distance between images, which is the same as the section thickness, if every section, or a multiple of this if sections at a regular intervals, are used. X and Y should be identical, since width and length of digital camera image pixels are usually identical, the value has to be calculated from real dimensions on sections. To do so, the following procedure is recommended (Fig. 9): Section images from the image stack are visualized with the help of an OrthoSlice module (Fig. 9B). On one of the sections (histological) structures are determined which lie as far as possible - with regard to image width or length — from each other. Their distance - in parallel to image edges - is then measured with the crop editor. When the latter is activated, a frame appears at the edges of the section image. At activated interactive mode (arrow tool-in contrast to orthographic camera mode) edges of the box are grabbed at the nodes in their middle and shifted towards the predetermined structures on the sections. This causes changes of the min index and max index values in the image crop area of the crop editor. The difference between these two new values equals the distance between the measured structures in voxels (or pixels). Alternatively the voxel distance between to structures in the OrthoSlice can be measured by simply applying the Measuring module ( $2 \mathrm{D}$ line). The value given in the un-calibrated file gives the voxel distance since one model unit equals one voxel by default. Then the distance between the identical structures is measured in the original section (identical to the one used in the crop editor) by means of, for example, microscope and ocular micrometer (Fig. 9A). The $\mathrm{X} / \mathrm{Y}$-values are subsequently calculated by dividing the real distance (gained from the measurement on the microscope) by the number of pixels (voxels) lying between the two structures in the image stack (obtained from the crop editor measurement). The resulting value as well as the slice distance has to be inserted in the respective fields of the crop editor voxel size line (Fig. 9E). 
2.6 Slice alignment and final dimension adjustment. The first actual processing step in $A M I R A$ is bringing section images of the image stack into congruency by rotation and translation (shifting). This

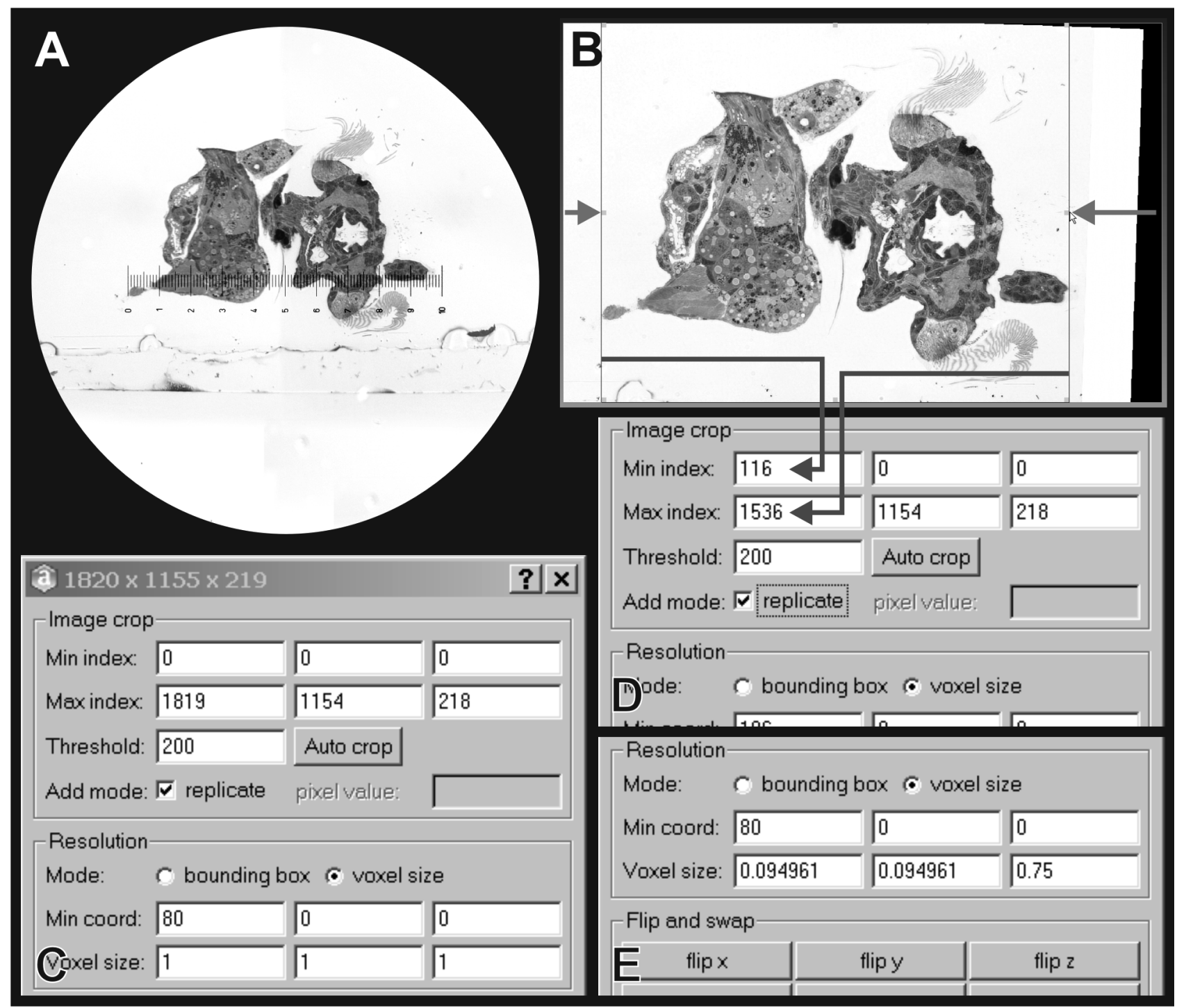

Figure 9. Calibration of the dimensions with the help of the crop editor with the example of a Patella caerulea larva (horizontally sectioned, $0.75 \mu \mathrm{m}$ section thickness, glutaraldehyde-osmium tetroxide fixation, Spurr's resin embedding, methylene blue-azurII dye, same specimen as Figs 12, 15, 16 and 17). A. Internal circle stands for the field of vision of a compound microscope with an ocular micrometer for measuring the same distance as in B. with the crop editor. B. Slice visualized with Axial OrthoSlice. Alignment has been performed previously. Edges of the crop editor box have been shifted towards the specimen from left and right for the distance indicated by the length of the red arrows. C. Part of the crop editor with unchanged (un-calibrated) settings. D. Part of the crop editor with changed setting after shifting the edges of the crop editor box towards the specimen. Values change according to the position of the box' edges in B (blue arrows). E. Part of the crop editor with new voxel sizes calculated from the measurements obtained herein: In the present example, the distance measured by A. is $134.9 \mu \mathrm{m}$. This equals 1,420 voxels. The result of dividing the former by the latter value is about 0.095 - the new $\mathrm{X}$ (first column) and $\mathrm{X}$ (second column) voxel dimension. The $\mathrm{Z}$ (third column) dimension is represented by section thickness (0.75). The new model unit equals $1 \mu \mathrm{m}$ because this was chosen as measurement dimension unit (A. and slice thickness). 
alignment process generally bears some perfidies. If there are no stable (external) references, one has to refer to histological structures as such. This may lead to major artificial twisting and dislocations in portions of the specimen. This problem may be explained with the help of a theoretical example, the case of a banana: How could one achieve banana shape by simple alignment of individual slices without a priori knowledge of the shape? In practice it is not as problematic as in this extreme example, because inside of biological specimens there typically are several different components, such as organ systems, that provide a reciprocal control. In case of resin embedding, the section (block) edges and also sectioning artefacts like scars can be very useful for alignment (Fig. 10). If the block has been sectioned vertically $\left(+/-90^{\circ}\right)$ to the block axis - relating to the mould shape as suggested above (Fig. 4A) - the block's bottom represents a stable reference plane. This means that the distance of the specimen to the block's bottom section edge remains the same for all sections. If, as recommended above, the specimen has been placed at the mould/block's bottom; this block surface is close to the specimen and provides a highly useful reference. If present, normally unintended scratches represent an addition alignment aid. In contrast to the block's bottom, the latter can be used for limited parts of the series because of knife position changes during sectioning. Both the block's bottom and scratches work perfectly for rotation alignment. The block's bottom over the whole series and scratches in certain areas can be used for translation alignment in one axis. The translation alignment of the perpendicular axis must be simply done according to congruency of specimen structure; however, it can be controlled by images taken from the embedded specimen prior to sectioning (Fig. 11A). The alignment result can be compared with the image by either moving a Coronal OrthoSlice (Fig. 12A: co) through the specimen to get a rough estimation of the shape achieved by alignment or by displaying a final surface visualization (see below).
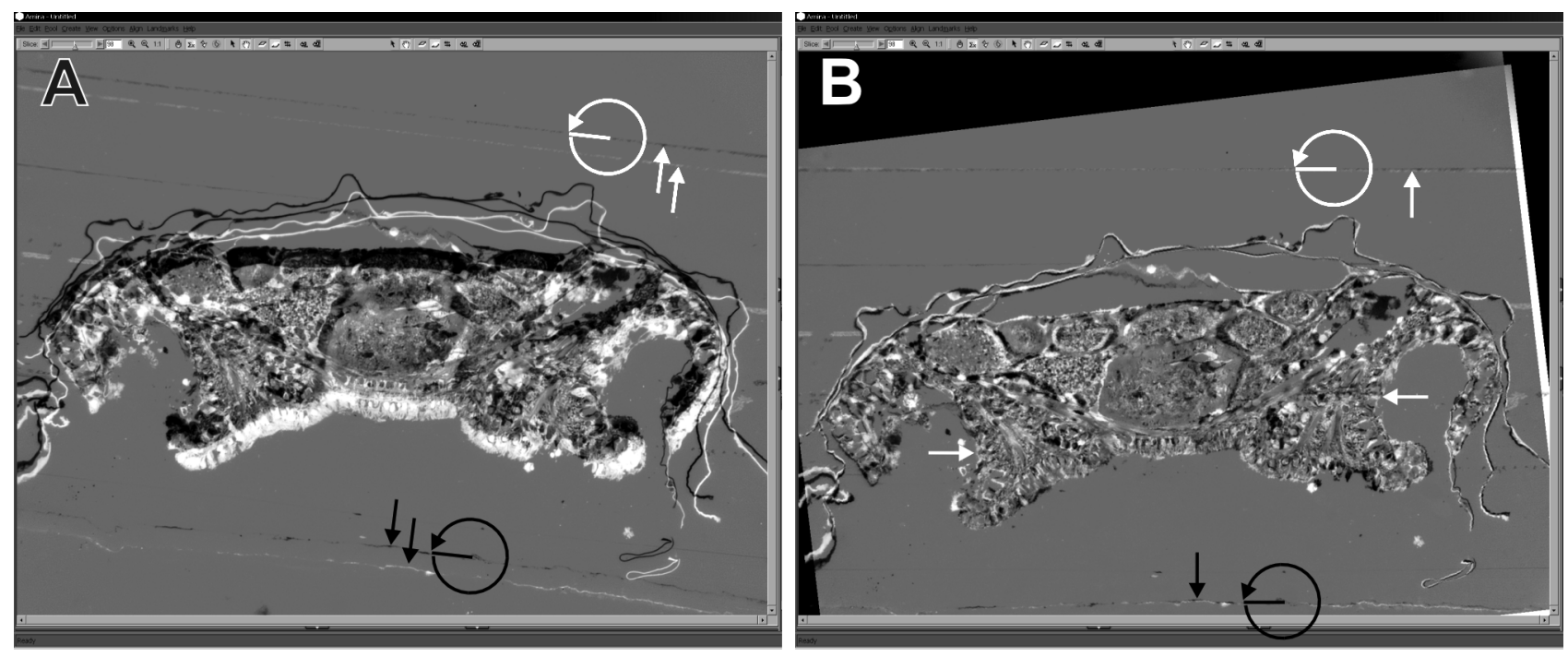

Figure 10. Section alignment with the alignslices module by utilizing a block edge (block/mould bottom, blue arrows and circle with arrow) and scratches (red arrows and circle with arrow) for translation (blue and red arrows) and rotation (circles with arrow) exemplified by the monoplacophoran Micropilina minuta (formaldehyde fixation, Araldite embedding, cross sectioned, $2 \mu \mathrm{m}$ section thickness, methylene blue-azurII dye, same specimen as Fig. 13). A. Unaligned sections. B. Aligned sections. Each edge of the section at the block's bottom positioned slightly above the margin of the window. Horizontal white arrows indicate the shifting direction that can only be adjusted according to congruency of specimen structure. 
The procedure in AMIRA is carried out with the alignslices module (Fig. 10). In practice it makes sense to begin with applying the align all slices function with the default Least-squares alignment mode, to have aligned all slices automatically. When this is applied, adjacent sections are shifted and transformed towards each other to minimize differences in brightness and contrast. If sections are of good quality and bear contrast rich areas, aligning all slices works really well and relatively little correction work by hand is necessary. Remaining problems mostly concern minor errors in rotation, which add up over longer distances. These errors can be controlled or adjusted with the help of the bottom edge of the sections or scratches (see above). For final alignment by hand it is very helpful to place the bottom edge of the sections slightly above the lower edge of the alignslices module window and to keep both edges parallel (Fig. 10B). Accordingly, this position needs to be kept fixed over the whole series. For hand alignment it is important to understand that it is not a single slice that is moved relative to all other slices. Instead the whole portion of the series lying anterior or posterior to this slice is moved together with this slice.

After completing alignment it might happen that the specimen does no longer fit into the volume of the image stack, which has initially been simply defined by the pixel numbers of the section images ( $\mathrm{X}$ and $\mathrm{Y}$ ) and the number of section images ( $\mathrm{Z}$ ) used. This is caused by shift of specimen portions required for alignment (Fig. 11, see also above for the "banana" example). In this case, there is a tool offered to enlarge the $\mathrm{X}$ and/or Y values prior to the last alignment step, the recalculation (resample in the alignslices menu) of the final images position. This function was already present in version 3.1.1, but it was unreliable and could be very tricky when applied in combination with the crop editor only. In version 4.1.1 it works well. Generally one should consider the problem of file size also for this procession step, since enlargement of dimension leads to enlargement of file size as well. If such an enlargement is necessary - the general shape of a specimen is mostly known — one should take this into account when initially planning the resolution of the image stack.

More often, however, after alignment is done, the image stack is larger in $\mathrm{X}$ and/or $\mathrm{Y}$ dimension than the specimen, i.e., the there is excess space that can be removed without deleting any part of the specimen. This is done with the crop editor - again for the purpose of reducing file size. First one moves through the stack by displaying an Axial OrthoSlice to detect the sites where the specimen is
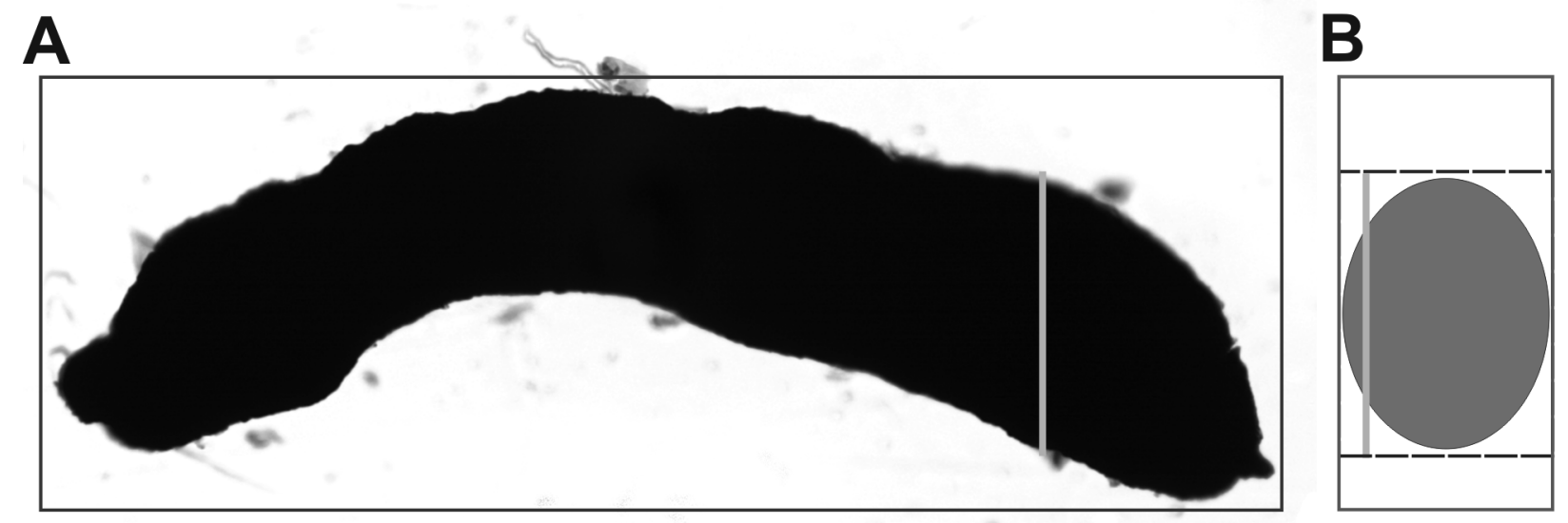

Figure 11. Demonstration of the problem if dimensions of the image stack file have to be enlarged in the course of section alignment exemplified by a small solenogaster (same specimen as in Figs 2, 3 and 4). Length of red vertical lines give required width of section images. Height of the green rectangles give required final X or $\mathrm{Y}$ dimension. A. Image of the specimen prior to sectioning (see Fig. 12). B. Theoretical relative dimension of a section at the position of the red vertical line in A. 
closest to the side. After activating the crop editor tool, similarly as for calibration (compare Fig. 9B), in orthographic camera mode one can activate the interactive mode (arrow tool) and grab the box at the sides or at the corners. The edges of the box can then be shifted from either side towards the specimen. By applying OK in the crop editor excess material is cropped from the image stack. The results of alignment, calibration and cropping can be visualized and controlled by displaying the image stack file with three perpendicular OrthoSlices (Fig. 12A).

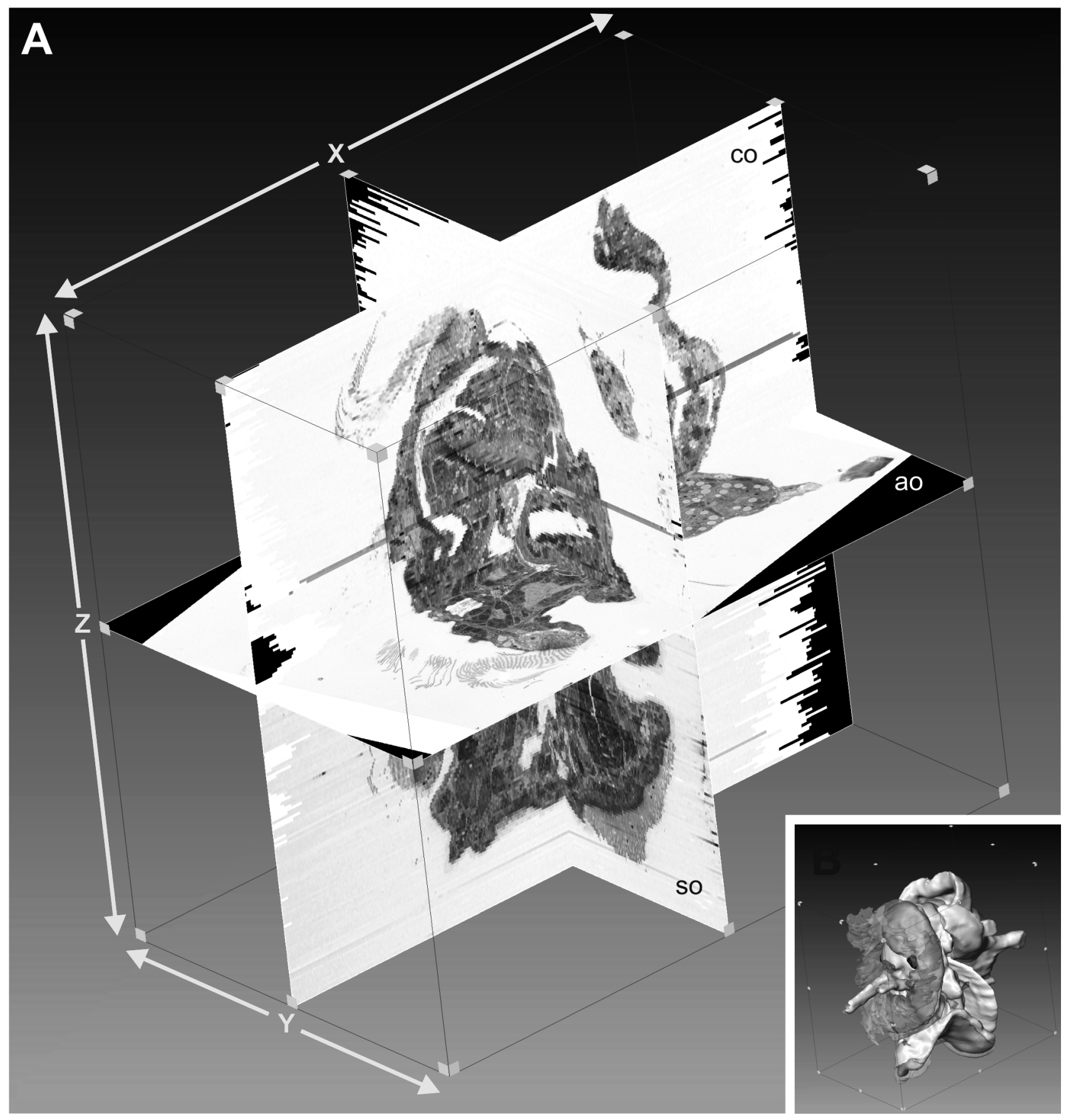

Figure 12. A. Aligned, cropped and calibrated image stack file visualized by three perpendicular OrthoSlices with three axes (red letters and arrows) of a Patella caerulea larva (same specimen as Figs 9, 15, 16 and 17). B. Surface rendering of the same specimen as A. at lower magnification to compare the actual shape of the specimen. ao, Axial OrthoSlice; co, Coronal OrthoSlice; so, Sagittal OrthoSlice. 
2.7 Labelling of structures (image segmentation). Labelling of structures is by far the most timeconsuming step of the whole procedure. It can - depending on individual preferences-be performed with a graphic tablet (e.g., CTE-440, Wacom Co., Ltd., Kita Saitama-Gun, Japan) or with a conventional modern computer mouse. Working with a graphic tablet requires practice to be efficiently applied. State of the art (laser) optical mice with at least three buttons can facilitate efficient working as well.

AMIRA provides instruments like the magic wand that theoretically could be used to do it automatically - even in $3 D$ - simultaneously for all sections. This would be based on brightness/contrast difference. But in practice - since we do not have such differences in brightness/contrast for most structures - this must be done by hand.

It is carried out with the Image Segmentation Editor. A separate image stack file (LabelField) is created with dimensions identical to the original image stack file. In the editor identical sections of both files are always displayed at once, with the LabelField image being displayed above the original image. One selects areas of the original images with different tools (e.g., brush, lasso or magic wand) and attributes these to Materials (e.g., organs). This information is saved in the LabelField file.

Segmentation should be nearly exclusively carried out with the XY $2 D$ viewer of the Image Segmentation Editor. This corresponds to the original section planes. There would be also the $X Z$ and $Y Z 2 D$ viewers (available via the 4-viewer mode) that give "virtual" sections normal to the original section planes, for labelling. The latter may be more helpful for the segmentation procedure of data sets of initially perfectly aligned "virtual" sections (from e.g., CLSM, CT), than for "physical" sections. Unfortunately, the 4-viewer mode, which is hardly useful for the purpose treated here, is opened by default in the newest version of $A M I R A$. This, in addition to other new default settings, is somewhat uncomfortable and may reflect the intention of the AMIRA developers to weight "virtual" section segmentation higher than "physical" section segmentation, a trend that hopefully does not continue.

There are strategies to reduce the effort at image segmentation. It is very important to make use of the interpolate (Image Segmentation Editor-selection menu) function (Fig. 13). For example, one should label only part of the images as a first step, such as the body surface of every third section. This is followed by interpolating in between these previously labelled sections (Fig. 13A-H). By interpolation intermediate selections are created that have intermediate size and shape approximating those of the previously created anterior and posterior located selections. Thus, by interpolation the original images are not consulted at all. This function simply relies on the idea that intermediate structures have intermediate size and shape too. Accordingly, the results need to be controlled for all automatically labelled sections. In many cases the results are satisfying but often need to be additionally corrected (Fig. 13J-K).

The suitability of structures for interpolation differs. Structures that are voluminous and roundish are much better suited than thin, flat or elongated ones. Problems caused by the structures of the latter kind can be bypassed by a variety of tricks, such as interpolating different parts of structures after each other (similar to Fig. 14E-G). Interpolation is not only useful to facilitate the segmentation process, but also to obtain relatively smooth material surfaces right away. Therefore, I recommendeven if interpolation is more laborious - never to label structures in consecutively slices by hand. If this is followed, errors that occurred during earlier work steps, such as bad alignment, can be found more easily and corrected if necessary. 
There are many other options to improve efficiency during image segmentation. The option to lock materials is very useful to avoid, for instance, that they, or parts of them, unintentionally become changed in case of overlapping selections. Also highly practical is the option to enlarge or reduce the size of selections even for all slices at once in single voxel dimension steps (Image Segmentation Editor-selection menu-grow-all slices). This may be used for tasks such as thickening delicate portions of Materials. Overall, there is broad range of tools available that allow a variety of strategies. A detailed treatment of such strategies, however, would go beyond the limits of the present paper. It only should be mentioned here that often it is very useful to established temporary Materials. The latter may be used for interpolating portions of Materials, or if locked, to set limits to other Materials. When not used anymore, such temporary Materials are merged with real Materials.

Throughout the detailed segmentation procedures, it is constantly necessary to consult the original section directly under the microscope. Except for very small specimens, the microscope provides more detailed information. This is mainly due to the availability of higher magnifications with better

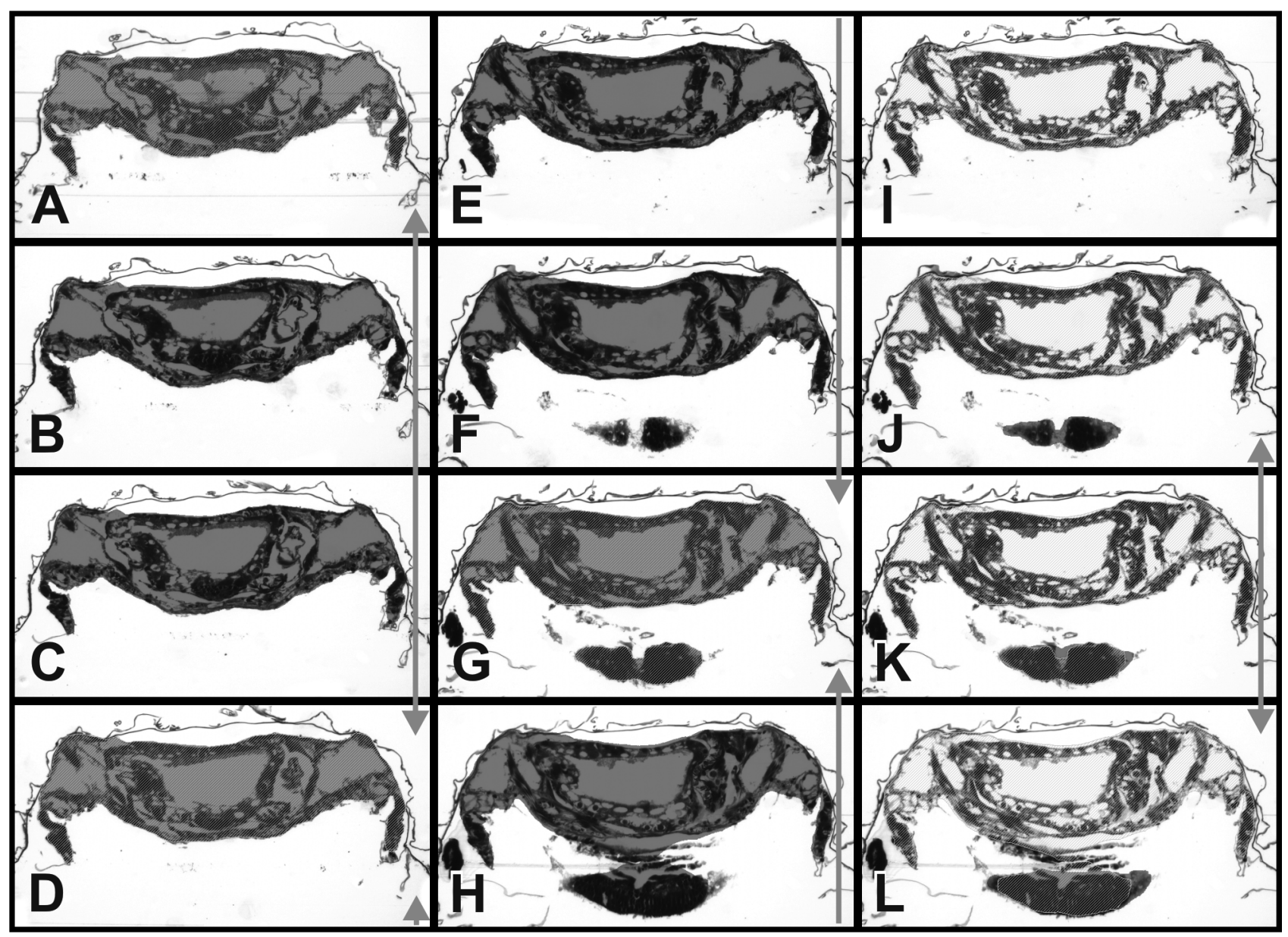

Figure 13. The general interpolation process exemplified by the monoplacophoran Micropilina minuta (same specimen as in Fig. 10). Blue arrows point to positions with highlighted selections prior to interpolation. A-H. Interpolation over the whole series from every third previously segmented section (A., D. and G.) demonstrated by some slices in the middle region. Interpolation result is shown in B-C, E-F and H. Note that anterior tip of the foot in F. was missimg because no comparable structure is present in D. I-L. Additional interpolation for the tip of the foot tip: It is labelled by hand in J. and L. with subsequent interpolation between these two slices. 
optical resolution and due to a minor degree to the original color information, which is lost in the grayscale Image Segmentation Editor images.

2.8 Generation of surfaces - surface rendering. Following image segmentation, surface rendering, the actual aim of the whole procedure, is carried out (Fig. 15). Initially, it mostly necessary - again for the purpose of reducing the amount of data to be manageable by the software - to apply the resample function (compute - resample) (Fig. 15A). This means a reduction of voxel resolution, four voxels in the plane of the sections are fused to one, if the default settings are used, which is recommended. The original resolution may only be required for delicate structures like nerves. When applying the surfgen module, the default setting add border is usually the right choice. If selected, the surface is closed anteriorly and posteriorly. There are also different Smoothing options. If unconstrained smoothing or constrained smoothing is selected, there are smoothing actions taking place - apparently applied to the slices - prior to actual surface generation. Experience tells that these smoothing operations are sufficient and make smoothing of labels in the Image Segmentation Editor (Labels-Smooth labels)

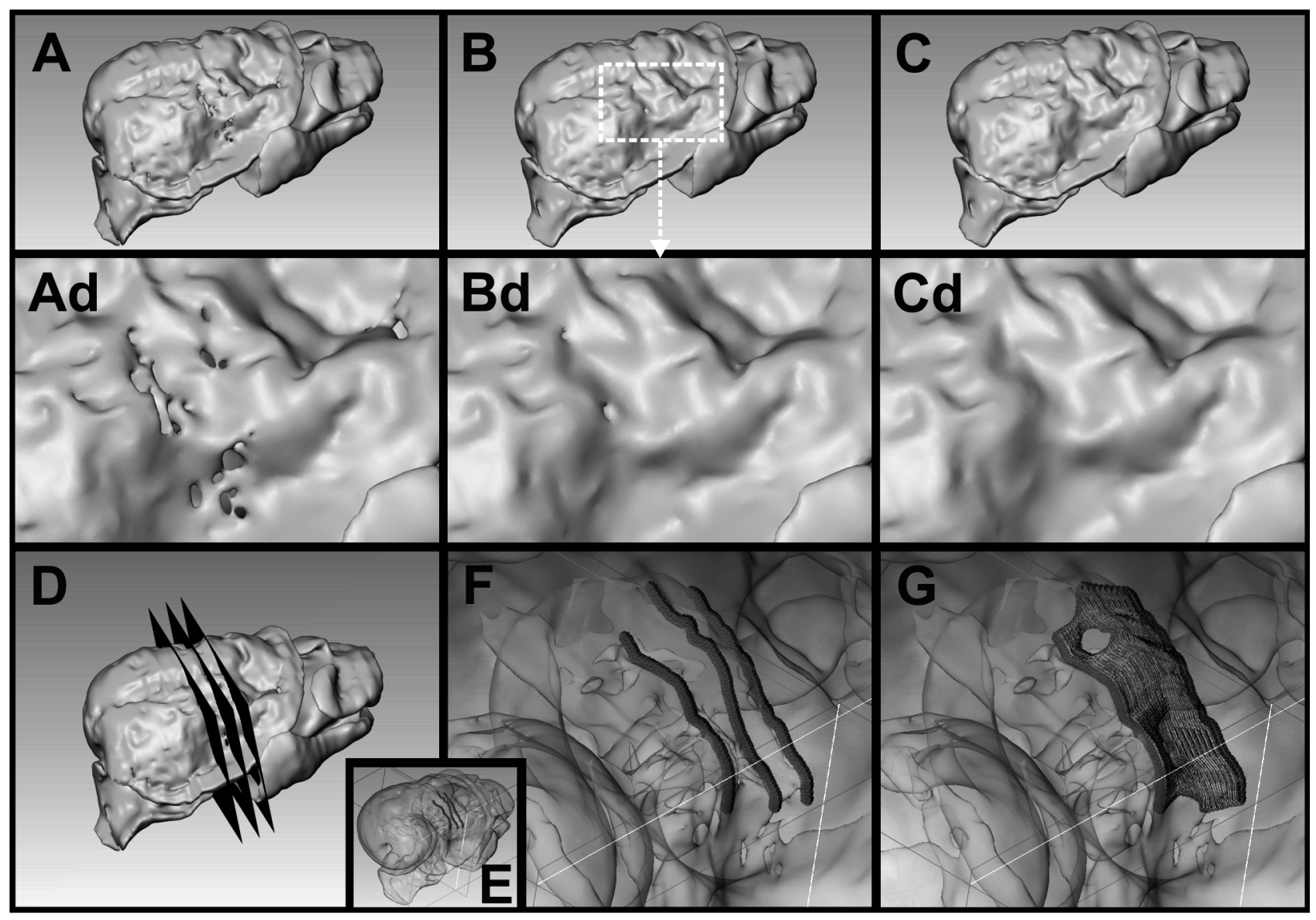

Figure 14. Correction works at the surface shown by the opisthobranch Haminoea navicula (juvenile, crosssectioned, $2 \mu \mathrm{m}$ thickness). A-C. Correction steps with gradual removal of errors such as holes etc. Upper row entire specimen. Middle row (Ad-Cd.) details with position given in B. D. Three Axial OrthoSlices displayed to locate the position of the problematic regions with regard to slice number. $\mathbf{E}-\mathbf{G}$. Specimen with transparent surface shown together with Selections in 3D. E-F. Roof of mantle cavity selected by hand in slices adjacent to positions with many errors. E. Total specimen. F. Detail of specimen. G. Same as F. after interpolation applied. 


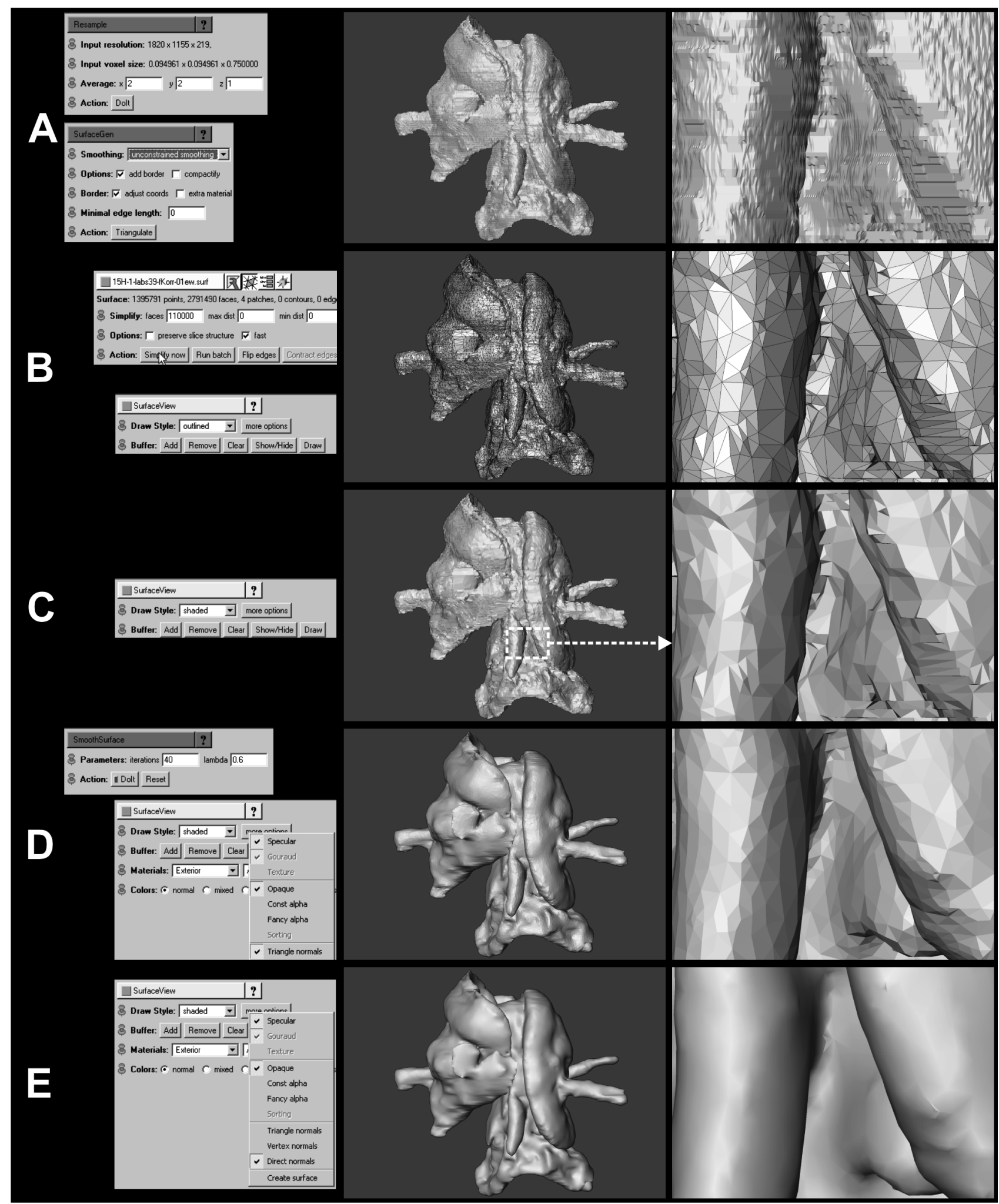

Figure 15. Steps in surface generation and refining demonstrated with a Patella caerulea larva (same specimen as Figs 9, 12, 16 and 17). Left columns shows the settings in AMIRA by screenshots of the respective tools. Middle (entire specimen) and right (enlarged part, position given in C.) show effects on the specimen surface. A. Initial result after resampling and applying SurfaceGen. B. Reduction of triangle number with the Surface Simplification Editor. Result shown with the outlined surface setting to visualize the triangular pattern of the surface. C. Same as B. but with shaded surface setting. D. Applying the SmoothSurface tool. E. Surface settings switched from Triangle normals to Direct normals. 
prior to surface generation redundant. Nearly always the most intense smoothing option, unconstrained smoothing, can be selected (Fig. 15A). Only for very delicate structures it might be useful to select none or constraint smoothing. Problems like lost connections for bent, string-like structures might also be avoided by omitting the resample function.

Surface rendering, which is performed with the surfgen module, called GMC in earlier programme versions, is based on the "generalizing marching cubes" algorithm. What is done by this procedure might be imagined as "stretching a skin consisting of triangles" over the edges of a body consisting of voxels." Accordingly, the geometrical properties of the surface file, reflected also in the file format (*.surf), are entirely different than the precursor, the LabelField file. At first, the surface is very large in file size and also in number of triangles. The latter has to be reduced with the Surface Simplification Editor (Fig. 15B) to make the surface manageable for further work steps. Like surface rendering, "simplifying" is a very intense task for the computer and it turned out that there is an upper limit in triangle number that can be dealt with. With the computers we used, this was about $4 \times 10^{6}$. To avoid an initial triangle number higher than this, one would have to go back to the LabelField and apply stronger resampling or reduce the Materials (see below). The final number of triangles might be adjusted to as many as 10,000-200,000 (Fig. 15B,C). The number is chosen according to the estimated complexity of the surface contained in the file. The resulting triangle number may still be a multiple of the minimal number actually required - the lowest triangle number where no loss of quality takes place. It, nevertheless, can be left like this, since there simply is no need to approximate this minimal number. File size of such surfaces is relatively low anyway.

Again, mainly for reasons of file size and digital processability, it often makes sense to separate the Materials prior to surface rendering (Fig. 16). They later can be visualized simultaneously (Fig. 16E).

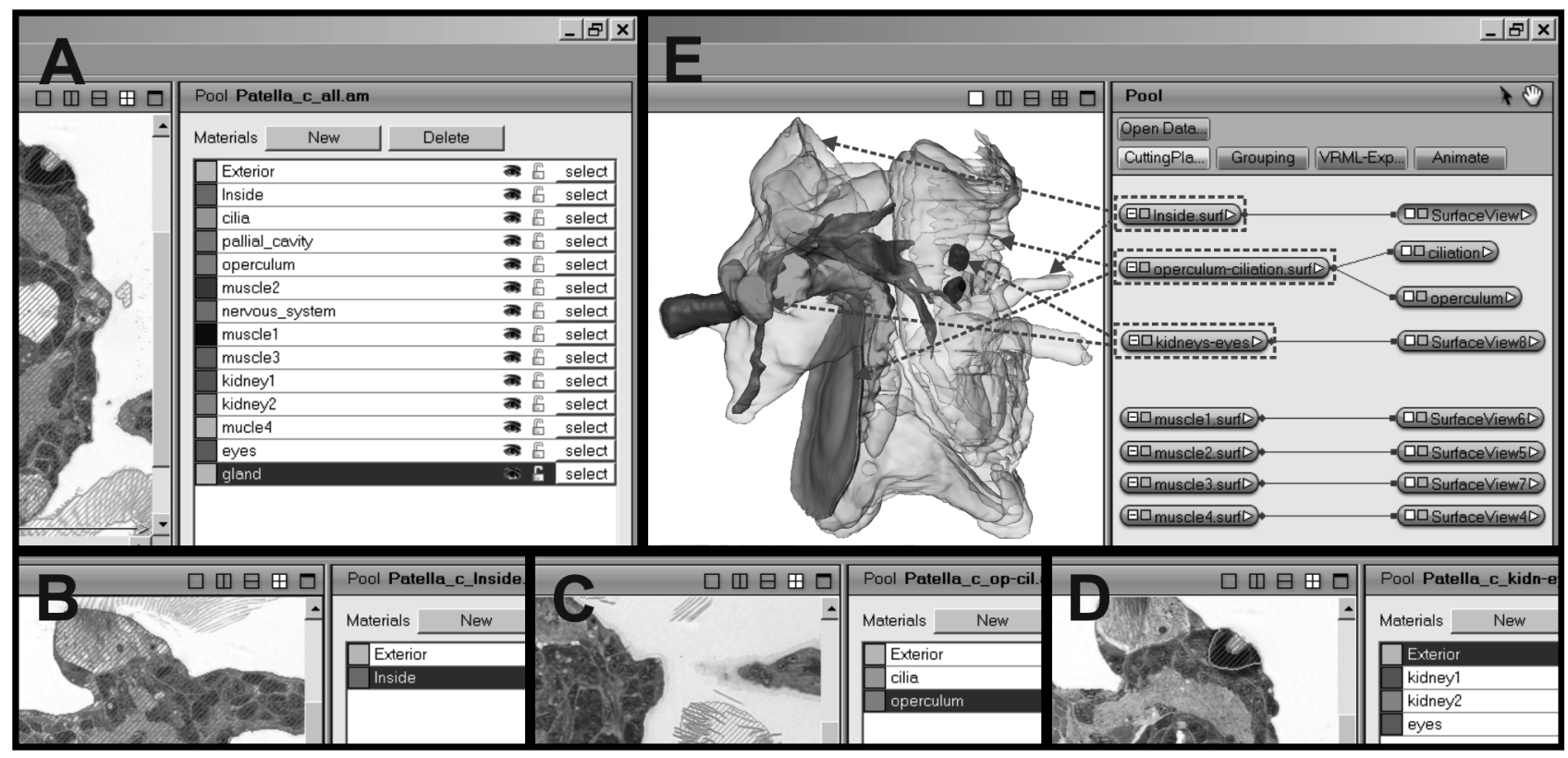

Figure 16. Steps in separating Materials in the Image Segmentation Editor by simultaneously displaying the resulting surfaces demonstrated with a Patella caerulea larva (same specimen as Figs 9, 12, 15 and 17). A. "Master" LabelField containing all segmented Materials. B-D. Separate LabelFields with different Materials deleted. Respective resulting surfaces files are highlighted with stippled rectangle in E. E. Surface visualization in the Amira Viewer (left) with surface files (right). Stippled arrows show position of respective surfaces resulting from files. 
To do so, certain Materials are removed from the LabelField originally containing all Materials and the resulting files are separately saved (Fig. 16B-D). Caution must be taken on the hierarchy of $M a-$ terials when performing this. When deleting a Material, it is assigned to (merged with) Exterior, the default Material surrounding all other Materials. The volume of a Material is still present inside the surrounding Material as a void space — and in the resulting surface — if simply deleted. First, this is a problem of file size (large total surface) and secondarily of visualization (internal surfaces displayed in transparent mode). Accordingly, internal structures must be merged with surrounding materials if it is not intended that their surface is contained in the resulting file. The easiest way to do this is renaming them like the surrounding Material. To give an example, when the external surface (might be called "inside" if default naming was used) of a specimen should be rendered, the "digestive_system" (or all components of this) should be renamed as "inside" (Image Segmentation Editor — click right onto material to be renamed). Subsequently, the surfaces of the new "inside" file and the separate file created for the "digestive_system" (simply deleting all other external Materials) can be displayed and manipulated at once in the Amira Viewer, where positions of surfaces from different files are perfectly correct relative to each other and identical as if originating from a single file.

The next step required is usually smoothing of the surfaces (compute-smooth surface; Fig. 15D). For the options to be set, a high value for iterations (e.g., 40) proved to be useful while lambda can be left at the default value (0.6). The value at Iterations only specifies the number of steps during the smoothing procedure. Despite of the high iterations value, the surface does not become seriously impaired by this process, like being noticeably distorted. The final adjustment for optimizing surface visualization is to set direct normals in the surfaceview (more options) (Fig. 15D,E). This is only a setting applied to the graphics card causing the triangular pattern of the surface to be hidden.

2.9 Correction works. The first surface gained is nearly always unsatisfactory. Although portions of the specimen may appear well reconstructed, other areas may show errors and disruptions. These could be holes were none are supposed to be, lost connections in delicate structures like nerves, Materials (from different *.surf files) that "break" through other ones or distinct-obviously artificial—curb extensions. Such "errors" need correction works (Fig. 14), which according to my experience are most efficiently carried out by going back to the Image Segmentation Editor. As a preparatory step, the slice positions of the sites to be corrected are located by displaying LabelField or image stack OrthoSlices together with the surface (Fig. 14D). Most of the corrections required can by carried out with the help of the interpolate function (see above). At slices posterior and anterior of the site to be fixed the respective parts of the specimen are selected (Fig. 14E,F) followed by subsequent interpolation between the selections. The interpolation result (resulting selection) (Fig. 14G) can be converted into the respective Material by applying $R$ (= replace), if it has a slightly different position or it is smaller, or $+(=A d d)$, if it is larger. If things are more complicated, it can be converted into a temporary Material (see above). Again, as stated above for the general segmentation procedure, there are numerous strategies, which cannot be explained in detail here.

A common problem is the appearance of holes in thin-layered Material portions (Fig. 14A). Here it often simply helps to thicken the layer. Similarly, thin, line shaped structures (e.g., nerves) that became disrupted in the surface, can be kept solid by thickening them. If Materials "break" through other ones, the layer of the overlaying Material has to be thickened. Accordingly, it must be admitted, that in many cases, just for the purpose of producing a satisfying surface, "artificial" labelling 
has to be done that is not really justified from the slice structures. However, with regard to total inaccuracies, like those resulting from procedures like interpolation, such "artificial" adjustments usually remain at a negligible level.

Obviously, there is a lot of personal interpretation involved in the whole process. My own attitude when I do this is: I am modelling a specimen - after a template, the image stack. Nevertheless, in the end the results become fairly realistic. This could be criticized as bad scientific practice. However, it must be noted that this was the same when specimen were reconstructed by hand prior to the days of computer processing. Smoothing corrections were applied no less intensely as described for the present method. At this point, the importance of section quality must be emphasized. Quality is generally much higher in resin sections than in, for example, paraffin sections. Such sections bear many more problems, for instance distortions or dislocations and losses of portions. With regard to computer reconstruction this would cause much more effort with less satisfying results.

During corrections works as given above, surfaces must be generated repeatedly, to control the segmentation results. In total, the correction works can take days and make up a major part of the whole computer reconstruction procedure.

One specific problem of surface generation in AMIRA must be mentioned here: There is no way to avoid that portions of a single material merge at positions where they are not supposed to. A typical

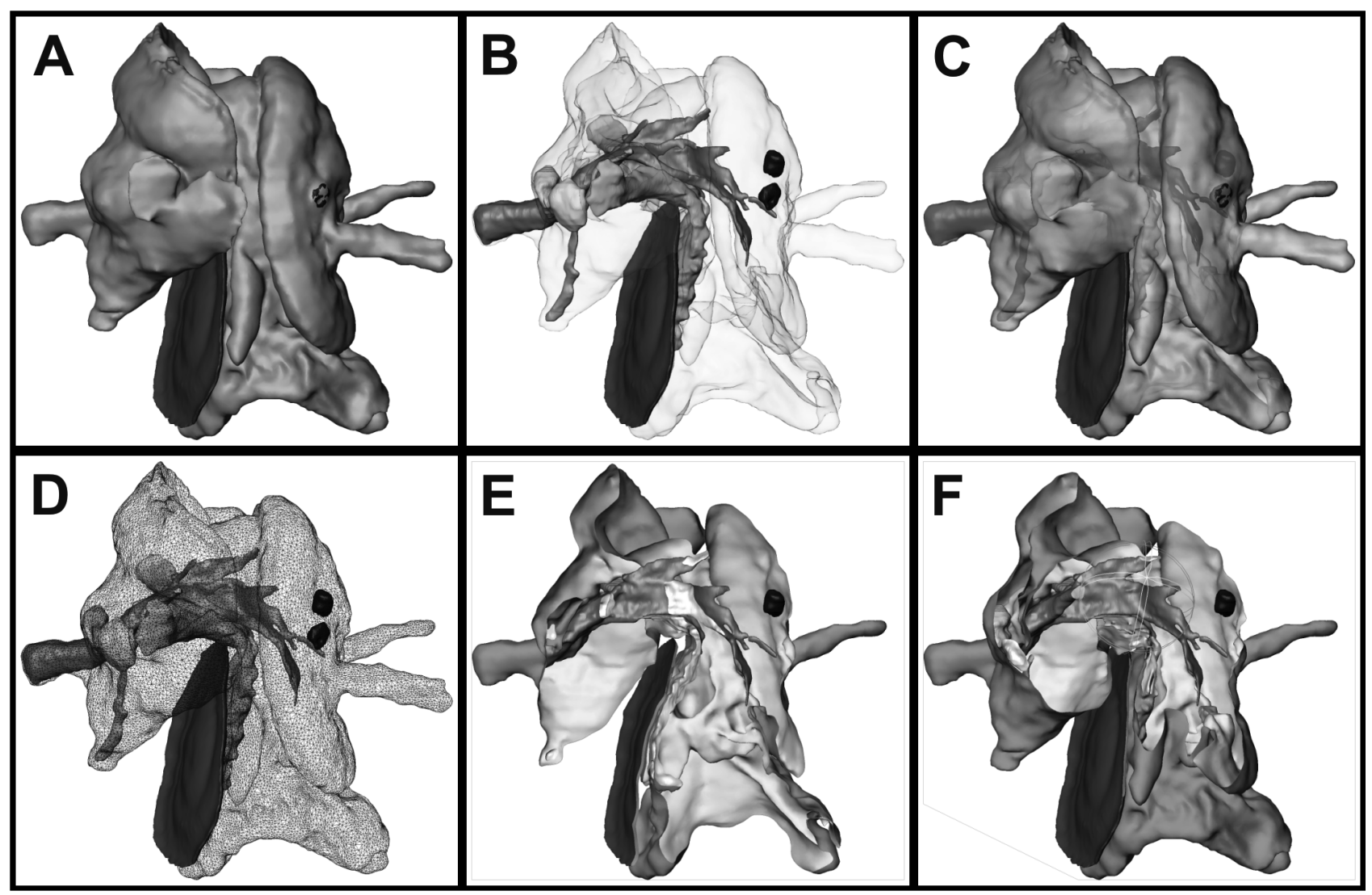

Figure 17. Examples of surface visualization in AMIRA demonstrated with a Patella caerulea larva (same specimen as Figs 9, 12, 15 and 16). A. Specimen external surface shaded. B-C. Different transparency intensities for the external surface. D. Wire frame like transparent outlined mode for the external surface. E. Same as A. with parts of the specimen made invisible by applying an intersect. F. Same as E. with changed angle (rotate) and position of the intersect. 
case in the field of microanatomy would be represented by gut loops that lie adjacent to each other. There is no way to separate such gut loops if they belong to the same Material and as a consequence there is no surface formed between them. There are only indentations formed between the loops, which by smoothing become even less pronounced. There may be ways to bypass this problem. Theoretically, one might initially create different Materials for individual loops, delete the unintended formed border surfaces between the loops (in contrast to external adjacencies) with the Surface editor and fuse the surfaces to a single surface Material. The latter options, however, do not seem to be easily available in $A M I R A$ so far. Hence, such goals and strategies might be matter of developing solutions to procedures with $A M I R A$ or similar software systems in the future.

Also mentioned should be the "staircase" effects. These appear if slice distances are too large (relatively high $\mathrm{Z}$ value — sections used too far from each other). They can be minimized with, for example, the InterpolateLabels function (Labelling). Since this is very intense task for the computer, this usually only can be applied to LabelFields that have already been resampled. The value 1 for intermediate slices resulting in doubling the slices is appropriate for the present purpose.

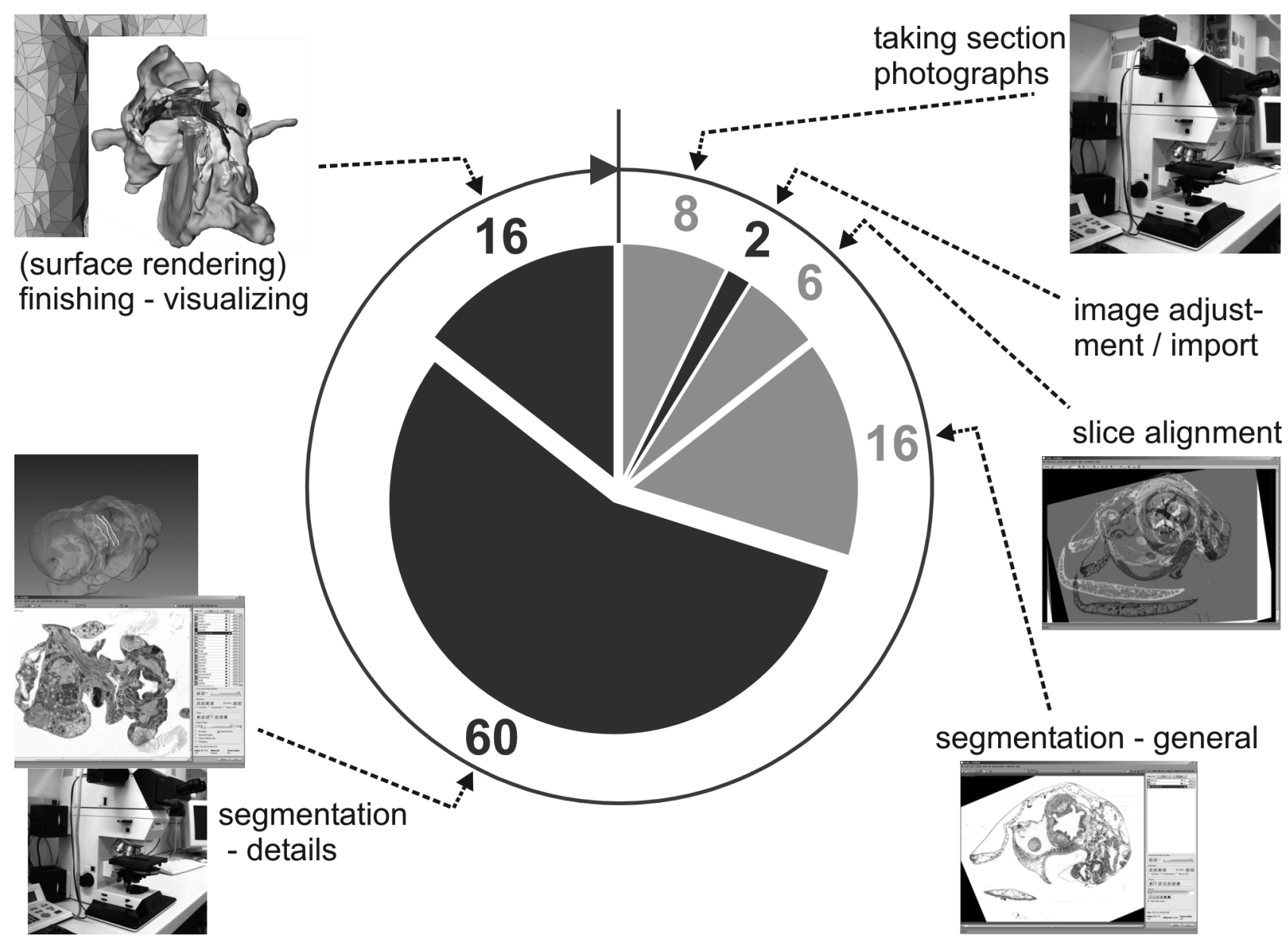

Figure 18. Expenditure of time based on my own estimate for an entire small molluscan specimen with good histological condition and processing of all major organ system without unexpected problems. Blue numbers give the estimated man-hours for individual processes. Bright blue marked processes can be carried out by an untrained person. Dark blue processes require an experienced worker. 
2.10 Final visualization. Once there are satisfactory surfaces, there are nearly endless possibilities of visualizing and displaying the specimen in AMIRA (Fig. 17). Among those are shaded (totally visible) surfaces or variable adjustable transparencies to show internal structures. There are different ways to display surfaces (rendering mode) like showing the geometry, with - for example - the triangles. Also, pieces could be "cut" off from the specimen by intersects to allow viewing internal components. AMIRA provides a variety of additional visualization modes, including stereo pairs (readily available in version 4.1.1) and highly sophisticated options coming with the VR Pack extension.

There is also an option to change surface colors, which is relatively hidden in the Parameter Dia$\log$ (materials-Material to be changed - color — click the color box on bottom right of the dialog window). 3D surfaces, like other visualization results (e.g., OrthoSlices), can be directly displayed in $A M I R A$ on the screen, whereby a variety of manipulation options, like rotation, change of magnification or change from orthographic to perspective view, to name only few, can be applied.

There are numerous ways to make use of such results for purposes other than directly displaying them with AMIRA. One can take 2D images, which basically are screenshots taken from the Amira Viewer window (part). These are valuable for all kinds of purposes, among these scientific publication images (e.g., Ruthensteiner 2006; Ruthensteiner et al. 2007; Ruthensteiner \& Stocker in press). It is also possible to create movie files. These could be, for instance, rotating 3D models to be displayed with a digital projector for presentations (scientific meetings, web).

Another option is transferring surface models/geometries to other 3D software systems. The popular*.pdf format offers the opportunity to display interactive 3D models directly, which can even be used for scientific publications (Barnes \& Fluke 2008). Ruthensteiner \& Heß (in press) introduce this format to biology and give a detailed description on how to transfer AMIRA surface meshes to the Adobe *.u3d format and embed them in *.pdf files to serve for publications (Baeumler et al. 2008).

2.11 Effort involved - duration of operating procedures. An important question is: How long does the whole graphical procedure take? Figure 18 shows the steps of the whole procedure in clockwise direction with estimated duration expressed in working hours. This is only a personal rough estimation for a specimen with few problems (good histology) for full adult anatomy (all organ systems). This is summing up to 108 working hours, which is nearly three weeks of work. According to this estimate, less than a third in total can be done by an untrained person, like a student helper. The other steps must be carried out by someone who is experienced or who is gathering experience as it happens in the course of a master/doctoral thesis.

\section{Acknowledgements}

I thank teachers and colleagues (e.g., H. Hilgers, W. Klepal, G. Pass, K. Schaefer, M. Walzl) of the old days at the zoological institute of the University of Vienna, who supplied valuable knowledge and discussions and histological techniques. G. Haszprunar's interest represented an important background in establishing the methods. The GeoBio-Center of the LMU Munich provided a license of $A M I R A$. I am particularly grateful to S. Schopf and C. Brossmann for their devotion to initially set up the array in AMIRA. Screenshots of AMIRA shown with kind permission of H.-C. Hege (ZuseInstitut Berlin). V. Schröpel helped in reconstruction of the Patella larva. Many thanks to D.L. Geiger for the invitation to join the symposium and the opportunity to publish this somewhat unusual 
paper in this volume. Thanks to R. Golding and C.E. Thacker for valuable comments and improving the English. Last not least I thank E. Lodde for assistance at a variety of procedures.

\section{References}

Adam, H. \& Czihak, G. (1964) Arbeitsmethoden der makroskopischen und mikroskopischen Anatomie. Gustav Fischer Verlag, Stuttgart, 583 pp.

Barnes, D.G. \& Fluke, C.J. (2008) Incorporating interactive 3-dimensional graphics in astronomy research papers. New Astronomy, in press. Also available from arXiv:0709.2734. http://astronomy.swin.edu.au/s2plot/3dpdf/ (accessed 8 January 2008).

Baeumler, N., Haszprunar, G. \& Ruthensteiner, B. (2008) 3D microanatomy of Omalogyra atomus (Philippi, 1841) (Omalogyridae, Gastropoda, Mollusca). Zoosymposia, 1, 101-118.

Bennet, H.S., Wyrick, A.D., Lee, S.W. \& McNeil, J.H. (1976) Science and art in preparing tissues embedded in plastic for light microscopy, with special reference to glycol methacrylate, glass knives and simple stains. Stain Technology, 51, 71-97.

Blumer, M.F.J., Gahleitner, P., Narzt, T., Handl, C. \& Ruthensteiner, B. (2002) Ribbons of semithin sections: an advanced method with a new type of diamond knife. Journal of Neuroscience Methods, 120, 11-16.

Böck, P. (1984) Der Semidünnschnitt. JF Bergmann Verlag, Munich, 172 pp.

Böck, P. (Ed. 1989) Romeis-Mikroskopische Technik. $17^{\text {th }}$ Edition. Urban und Schwarzenberg. Munich-Vienna-Baltimore, $697 \mathrm{pp}$.

Bohn, J.M. \& Heinzeller, T. (1999) Morphology of the bourgueticrinid and isocrinid aboral nervous system and its possible phylogenetic implications (Ecinodermata, Crinoidea). Acta Zoologica, 80, 241-149.

Bozzola, J.J. \& Russell, L.D. (1998) Electron Microscopy: Principles and Techniques for Biologists. $2^{\text {nd }}$ Edition. Jones \& Bartlett Publishers Inc., U.S., Boston, 670 pp.

Brandt, R., Rohlfing, T., Rybak, J., Krofczik, S., Maye, A., Westerhoff, M., Hege, H.-C. \& Menzel, R. (2005) Threedimensional average-shape atlas of the honeybee brain and its applications. The Journal of Comparative Neurology, 492, 1-19.

Clendenon, J.L., Byars, J.M. \& Hyink, D.P. (2006) Image processing software for 3D light microscopy. Nephron Experimental Nephrology, 103, e50-e54.

Dietrich, H.F. \& Fontaine, A.R. (1975) A decalcification method for ultrastructure of echinoderm tissue. Stain Technology, 50, 351-354.

Eisenmann, E.A. \& Alfert, M. (1982) A new fixation procedure for preserving the ultrastructure of marine invertebrate tissue. Journal of Microscopy, 125, 117-120.

Emery, D.G. (1992) Fine structure of olfactory epithelia of gastropod molluscs. Microscopy Research and Technique, 22, $307-324$.

Fiala, J.C. (2005) Reconstruct: a free editor for serial section microscopy. Journal of Microscopy, 218, 52-61.

Geiger, D.L., Marshall, B.A., Ponder, W.F., Sasaki, T. \& Waren, A. (2007) Techniques for collecting, handling, preparing, storing and examining small molluscan specimens. Molluscan Research, 27, 1-50.

Glauert, A.M. \& Lewis, P.R. (1998) Biological Specimen Preparation for Transmission Electron Microscopy. Practical Methods in Electron Microscopy. Volume 17. Glauert, A.M. (Ed.), Portland Press, London, 315 pp.

Haas, A. \& Fischer, M.S. (1997) Three-dimensional reconstruction of histological sections using modern product-design software. The Anatomical Record, 249, 510-516.

Haszprunar, G. (1988) Anatomy and relationship of the bone-feeding limpets, Cocculinella minutissima (Smith) and Osteopelta mirabilis Marshall (Archaeogastropoda). Journal of Molluscan Studies, 54, 1-20.

Henry, E.C., (1977) A method for obtaining ribbons of serial sections of plastic embedded specimens. Stain Technology, $52,59-60$.

Huijsmans, D.P., Lamers, W.H., Los, J.A. \& Strackee, J. (1986) Toward computerized morphometric facilities: a review of 58 software packages for computer-aided three-dimensional reconstruction, quantification, and picture generation from parallel serial sections. The Anatomical Record, 216, 449-470. 
Kiernan J.A. (2001) Histological and Histochemical Methods. Theory and Practice. $3^{\text {rd }}$ Edition. Reed Educational and Professional Publishing Ltd, Oxford, 502 pp.

Luft, H. (1961) Improvements in epoxy resin embedding methods. Journal of Biophysical and Biochemical Cytology, 9 , 409-414.

Neusser, T.P., Heß, M., Haszprunar, G. \& Schroedl, M. (2006) Computer-based three-dimensional reconstruction of the anatomy of Microhedyle remanei (Marcus, 1953), an interstitial acochlidian gastropod from Bermuda. Journal of Morphology, 267, 231-247.

Nielsen, C., Haszprunar, G., Ruthensteiner, B. \& Wanninger, A. (2007) Early development of the aplacophoran mollusc Chaetoderma. Acta Zoologica (Stockholm), 88, 231-247.

Page, L.R. (1998) Sequential developmental programmes for retractor muscles of a caenogastropod: reappraisal of evolutionary homologues. Proceedings of the Royal Society of London, Series B, 265, 2243-2250.

Pass, G. (1985) Gross and fine structure of the antennal circulatory organ in cockroaches (Blattoidea, Insecta). Journal of Morphology, 185, 255-268.

Pieper, S. Lorensen, B. Schroeder, W. Kikinis, R. (2006) The NA-MIC Kit: ITK, VTK, pipelines, grids and 3D slicer as an open platform for the medical image computing community. Proceedings of the IEEE International Symposium on Biomedical Imaging, 1, 698-701.

Reid, N. \& Beesley, J.E. (1991) Sectioning and Cryosectioning for Electron Microscopy. Practical Methods in Electron Microscopy, Volume 13. Glauert, A.M. (Ed.), Elsevier, Amsterdam, xviii + 322 pp.

Richardson, K.C., Jarett, L. \& Finke, E.H. (1960) Embedding in epoxy resins for ultrathin sectioning in electron microscopy. Stain Technology, 35, 313-323.

Rost, F.W.D. \& Oldfield, R.J. (2000) Photography with a Microscope. Cambridge University Press, Cambridge, 278 pp.

Ruthensteiner, B. (1997) Homology of the pallial and pulmonary cavity of gastropods. Journal of Molluscan Studies, 63, 353-371.

Ruthensteiner, B. (1999) Nervous system development of a primitive pulmonate (Mollusca: Gastropoda) and its bearing on comparative embryology of the gastropod nervous system. Bolletino Malacologico, 34, 1-22.

Ruthensteiner, B. (2006) Redescription and 3D morphology of Williamia gussonii (Gastropoda, Siphonariidae). Journal of Molluscan Studies, 72, 327-336.

Ruthensteiner, B. \& Heß, M. (in press) Embedding 3D-models of biological specimens in pdf-publications. Microscopy Research and Technique.

Ruthensteiner, B., Lodde, E. \& Schopf, S. (2007) Genital system development of Williamia radiata (Gastropoda, Siphonariidae). Zoomorphology, 126, 17-29.

Ruthensteiner, B. \& Schaefer, K. (1991) On the protonephridia and "larval kidneys" of Nassarius (Hinia) reticulatus (Linnaeus) (Caenogastropoda). Journal of Molluscan Studies, 57, 323-329.

Ruthensteiner, B. \& Stocker, B. (in press) Genital system anatomy and development of Ovatella myosotis by 3D computer visualization. Acta Zoologica (Stockholm).

Ruthensteiner, B., Wanninger, A. \& Haszprunar, G. (2001) The protonephridial system of the tusk shell, Antalis entalis (Mollusca, Scaphopoda). Zoomorphology, 121, 19-26.

Sätzler, K. (2001) 3D - Rekonstruktion von Serienschnitten. In: Plesser, T. \& Hayd, H. (Eds), Forschung und wissenschaftliches Rechnen, Beiträge zum Heinz-Billing-Preis 2000. GWDG, Goettingen, pp. 31-47.

Smaldon, G. \& Lee, E.W. (1979) A synopsis of methods for narcotisation of marine invertebrates. The Royal Scottish Museum Information Series, Natural History, 6, 1-96.

Stalling, D., Westerhoff, M. \& Hege, H.-C. (2005) Chapter 38. Amira - a highly interactive system for visual data analysis. In: Hansen, C.D. \& Johnson, C.R. (Eds), The Visualization Handbook. Elsevier, Amsterdam, pp. 749-767. 
Appendix 1. Protocols for histological chemicals with their application and specifications for computer software and hardware applied.

1. TEM fixation [see e.g., Glauert \& Lewis (1998) for safety precautions]

a. Marine invertebrates [modified from Eisenmann \& Alfert (1982)]

Primary fixative (4\% glutaraldehyde in $0.2 \mathrm{M}$ cacodylate buffer, $0.1 \mathrm{M} \mathrm{NaCl}, 0.35 \mathrm{M}$ sucrose $\mathrm{pH} 7.2-7.4)$ :

Buffer I:

Sodium cacodylate

$2.14 \mathrm{~g}$

$\mathrm{NaCl}$

$0.292 \mathrm{~g}$

Sucrose

$5.99 \mathrm{~g}$

Fill with distilled water to $50 \mathrm{ml}$. Adjust $\mathrm{pH}$ with $\mathrm{HCl}$ or $\mathrm{NaOH}$ solution to 7.2-7.4.

Final mixture (again adjust $\mathrm{pH}$ to $7.2-7.4$ with $\mathrm{HCl}$ or $\mathrm{NaOH}$ solution)

$25 \%$ glutaraldehyde

$2 \mathrm{ml}$

Buffer I

$10.5 \mathrm{ml}$

Secondary fixative ( $1 \%$ osmium tetroxide, in $0.2 \mathrm{M}$ cacodylate buffer, $0.3 \mathrm{M} \mathrm{NaCl}, \mathrm{pH} 7.2$ )

Buffer II:

Sodium cacodylate

$\mathrm{NaCl}$

$0.856 \mathrm{~g}$

Fill with distilled water to $20 \mathrm{ml}$. Adjust $\mathrm{pH}$ with $\mathrm{HCl}$ or $\mathrm{NaOH}$ solution to 7.2-7.4.

Final mixture:

$4 \%$ osmium tetroxide

$2 \mathrm{ml}$

Buffer II

$4 \mathrm{ml}$

Distilled water

$2 \mathrm{ml}$

Fixation procedure (if possible carried out at $4^{\circ} \mathrm{C}$ ):

Primary fixative

1-2 hours (or up to several days)

Buffer I : distilled water 1:1

$3 \times 15$ minutes

Secondary fixative

1-2 hours

Buffer II : distilled water $1: 1$

$3 \times 15$ minutes 
b. Fresh water invertebrates (e.g., Emery 1992)

Buffer (stock solution $0.02 \mathrm{M}$ )

Sodium cacodylate

$0.86 \mathrm{~g}$

Fill with distilled water to $200 \mathrm{ml}$. Adjust $\mathrm{pH}$ with $\mathrm{HCl}$ or $\mathrm{NaOH}$ solution to initially 8 or slightly above and later for the rinsing buffer (half concentration) and osmium tetroxide solution to 7.2-7.4.

Primary fixative [1.5\% glutaraldehyde in 0.01 (slightly lower) M cacodylate buffer]

$\begin{array}{lr}25 \% \text { glutaraldehyde } & 6 \mathrm{ml} \\ \text { Buffer } 0.02 \mathrm{M} & 47 \mathrm{ml} \\ \text { Distilled water } & 47 \mathrm{ml}\end{array}$

Adjust $\mathrm{pH}$ with $\mathrm{HCl}$ or $\mathrm{NaOH}$ solution to 7.2-7.4.

Secondary fixative (1\% osmium tetroxide in $0.01 \mathrm{M}$ cacodylate buffer)

$\begin{array}{lr}4 \% \text { osmium tetroxide } & 2 \mathrm{ml} \\ \text { Buffer } 0.02 \mathrm{M} & 4 \mathrm{ml} \\ \text { Distilled water } & 2 \mathrm{ml}\end{array}$

c. Terrestrial animals (e.g., Emery 1992)

Buffer (stock solution 0.1 M)

Sodium cacodylate $\quad 4.28 \mathrm{~g}$

Fill with distilled water to $200 \mathrm{ml}$. Adjust $\mathrm{pH}$ with $\mathrm{HCl}$ or $\mathrm{NaOH}$ solution to initially 8 or slightly above and later for the rinsing buffer (half concentration) and osmium tetroxide solution to 7.2-7.4.

Primary fixative (1.5\% glutaraldehyde in 0.05 (slightly lower) M cacodylate buffer)

$25 \%$ glutaraldehyde $6 \mathrm{ml}$

Buffer $0.1 \mathrm{M} \quad 47 \mathrm{ml}$

Distilled water $\quad 47 \mathrm{ml}$

Adjust $\mathrm{pH}$ with $\mathrm{HCl}$ or $\mathrm{NaOH}$ solution to 7.2-7.4.

Secondary fixative ( $1 \%$ osmium tetroxide in $0.05 \mathrm{M}$ cacodylate buffer)

$4 \%$ osmium tetroxide $\quad 2 \mathrm{ml}$

Buffer $0.1 \mathrm{M} \quad 4 \mathrm{ml}$

Distilled water $\quad 2 \mathrm{ml}$ 
Fixation procedure for freshwater and terrestrial animals (if possible carried out at $4^{\circ} \mathrm{C}$ ):

Primary fixative

1-2 hours (or up to several days)

Rinsing buffer (half conc. of stock solution)

$3 \times 15$ minutes

Secondary fixative

1-2 hours

Rinsing buffer (half conc. of stock solution)

$3 \times 15$ minutes

In general, it does not seem to affect histological quality substantially — at least it does not at the light microscopical level - if the material is left longer (up to a few weeks, cooled) in the primary fixative. This is important for field trips, where further processing of material is often impossible.

\section{Decalcification (following osmium tetroxide fixation)}

a. Ascorbic acid (1\% in $0.3 \mathrm{M} \mathrm{NaCl}$ solution, Dietrich \& Fontane 1975)

$\mathrm{NaCl}$

$0.82 \mathrm{~g}$

Ascorbic acid

$1 \mathrm{~g}$

Fill with distilled water to $100 \mathrm{ml}$.

b. EDTA (2\%, modified from Böck 1989)

EDTA (= Titriplex ${ }^{\circledR}$, Komplexon-III $\left.{ }^{\circledR}\right)$

$10 \mathrm{~g}$

Fill with distilled water to $100 \mathrm{ml}$. Dilute by stirring at $80^{\circ} \mathrm{C}$ and add drops of $\mathrm{NaOH}$ solution until $\mathrm{pH}$ is 7.2-7.4. Subsequently fill with distilled water to $400 \mathrm{ml}$ and adjust $\mathrm{pH}$ to 7.2 before filling up to $500 \mathrm{ml}$ with distilled water.

For both ascorbic acid and EDTA: treatment follows rinsing after osmium tetroxide during TEM fixation. Specimens are left in the solution over night or until the calcium is gone (could be checked with a polarizing system on a stereo microscope), while gently agitating (rotating). Specimen should be prevented from being exposed to light. Both treatments work for marine and freshwater animals. EDTA may be somewhat slower but gentler and can also be used for e.g., histochemical purposes. Specimen can be transferred to $30 \%$ acetone after decalcification and dehydrated for embedding as given below.

It should be mentioned that shell calcium of very delicate specimens often disappears if specimens are retained in the primary fixative or rinsing buffer following the primary fixative for a while (longer than a few hours) without special treatment.

\section{Resins}

Araldite:

Araldite M

$11.6 \mathrm{~g}$

Hardener (964B)

$9.98 \mathrm{~g}$

Accelerator (964C)

$0.46 \mathrm{~g}$

DBP

$1.4 \mathrm{~g}$ 
This represents a modification of the Araldite M protocol given by Glauert \& Lewis (1998). It has very good sectioning, but bad infiltration properties. It is recommended for conventionally fixed material. Polymerization: about 12 hours at $60^{\circ} \mathrm{C}$ with subsequent additional hardening for 6 hours at $95^{\circ} \mathrm{C}$.

Spurr's resin (= "SPURR's low viscosity resin", "ERL”):

$\begin{array}{lr}\text { ERL 4221D (ERL 4206) } & 10 \mathrm{~g} \\ \text { DER } 736 & 6 \mathrm{~g} \\ \text { NSA } & 26 \mathrm{~g} \\ \text { DMAE } & 0.4 \mathrm{~g}\end{array}$

This is a medium hard mixture (adjustment of hardness with DER, see Glauert \& Lewis, 1998). Because of its low viscosity it penetrates the tissue well. Polymerization does not always work as desired. It is/was regarded as highly toxic because of the ERL component. Recently Serva Electrophoresis (Heidelberg, Germany) launched a less toxic replacement (ERL 4221D), which works well. It is recommended for TEM fixed material. Polymerization: $12-24$ hours at $70^{\circ} \mathrm{C}$.

Epon:

$\begin{array}{lr}\text { “Epon” (Glycidether 100, C. Roth GmbH) } & 24 \mathrm{~g} \\ \text { DBA } & 15.5 \mathrm{~g} \\ \text { MNA } & 12.1 \mathrm{~g} \\ \text { DMP-30 } & 0.82 \mathrm{~g}\end{array}$

This corresponds to the $3: 2$ ratio of Luft's (1961) A and B mixtures and, thus, represents a relatively soft variant. This resin is also penetrating the tissue well and can be recommended for TEM fixed material. Polymerization: 24 hours at $60^{\circ} \mathrm{C}$.

All resins are thoroughly mixed by hand stirring. The specimens should/could be treated in a low pressure chamber ( $300 \mathrm{mb}, 20$ minutes) to facilitate mixing of components and force gas bubbles out. Mixed resins last for a couple of days if stored in the freezer (longer for Spurr's resin). Avoid water condensation at the resin surface when warming them for use (same for components)!

\section{Embedding procedure}

If resulting from TEM fixation, specimens are treated by steps of 30\%, 50\%, 70\%, 90\% and 100\% $(3 \times)$ acetone, each for $10(-20)$ minutes. If specimens have been conventionally fixed (typically stored in $\sim 75 \%$ ethanol), they initially may be totally stained with Safranin $(0.5 \%$ Safranin O in $80 \%$ ethanol, 10-20 minutes), in order not to lose them when they become transparent in the embedding resin. Subsequently they can either be rinsed in $80 \%$ ethanol or directly transferred to $90 \%$ acetone and further treated like TEM fixed material.

The pure acetone should be $\mathrm{H}_{2} \mathrm{O}$ free by "drying" with, for example, $\mathrm{CuSO}_{4}$. The pure acetone step is followed by a step of acetone : embedding resin 1:1 (overnight). This should be carried out with a small opening at the lid, allowing slow evaporation of the acetone. Agitation with a rotator is useful. Then specimens may be transferred to pure embedding resin (1-2 hours prior to placement in the 
moulds. Here (also when specimens are already in the moulds) treatment in a low pressure chamber ( $\sim 300 \mathrm{mb}, 1-2$ hours) may be helpful to facilitate penetration of tissue and force gas bubbles out of the surrounding resin. Controlling specimen orientation in the moulds before and after beginning of polymerization may be helpful.

\section{Section staining}

a. Methylene blue-azur II (= "Mallorys azur II-methylene blue" or "Richardson's stain"—-modified from Richardson et al. 1960)

$\begin{array}{ll}\text { Methylene blue } & 1 \mathrm{~g} \\ \text { Azur II } & 1 \mathrm{~g} \\ \text { Sodium tetraborate ("borax") } & 1 \mathrm{~g}\end{array}$

Fill with distilled water to $100 \mathrm{ml}$.

b. Toluidine blue

Toluidine blue $\mathrm{O}$ $1 \mathrm{~g}$

Sodium tetraborate ("borax")

$1 \mathrm{~g}$

Fill with distilled water to $100 \mathrm{ml}$.

Both mixtures represent stock solutions. For storage of different concentrations, syringes with attached filters are best suited.

\section{Specifications of software and hardware applied}

We started with software versions AMIRA 2.3 and 3.0 (TGS Europe, S.A., Merignac Cedex, France) and a PC (processor: Intel P IV 2.0A GHz, RAM: 1,536 MB, graphics card: Nvidia GF-4 Ti4400, operating system: Windows 2000).

AMIRA 3.1.1 (TGS Europe, S.A., Merignac Cedex, France) and AMIRA 4.1.1 (Mercury Computer Systems Inc., Chelmsford, MA USA) we used on a PC (processor: AMD K7 XP 3000+, RAM: 1,024 MB, graphics card: Sparkle SP8835XT-DT GF-FX 590, operating system: Windows XP professional edition). 